\title{
Cooperation and Competition Strategies in Multi-objective Shape Optimization - Application to Low-boom/Low-drag Supersonic Business Jet
}

\author{
Andrea Minelli*, Itham Salah el Din† Gérald Carrier ${ }^{\dagger}$ \\ ONERA - The French Aerospace Lab, F-92190, Meudon, France \\ Adrien Zerbinati; and Jean-Antoine Désidéri; \\ INRIA, Centre de Sophia Antipolis Méditerranée, 2004 Route des Lucioles, \\ BP 93, 06902 Sophia Antipolis Cedex, France
}

\begin{abstract}
Cooperation and competition are natural laws that regulate the interactions between agents in numerous physical, or social phenomena. By analogy, we transpose these laws to devise efficient multi-objective algorithms applied to shape optimization problems involving two or more disciplines. Two efficient strategies are presented in this paper: a multiple gradient descent algorithm (MGDA) and a Nash game strategy based on an original split of territories between disciplines. MGDA is a multi-objective extension of the steepest descent method. The use of a gradient-based algorithm that exploits the cooperation principle aims at reducing the number of iterations required for classical multi-objective evolutionary algorithms to converge to a Pareto optimal design. On the other hand side, the Nash game strategy is well adapted to typical aeronautical optimization problems, when, after having optimized a preponderant or fragile discipline (e.g. aerodynamics), by the minimization of a primary objective-function, one then wishes to reduce a secondary objective-function, representative of another discipline, in a process that avoids degrading excessively the original optimum. Presently, the combination of the two approaches is exploited, in a method that explores the entire Pareto front. Both algorithms are first analyzed on analytical test cases to demonstrate their main features and then applied to the optimum-shape design of a low-boom/low-drag supersonic business jet design problem. Indeed, sonic boom is one of the main limiting factors to the development of civil supersonic transportation. As the driving design for low-boom is not compliant with the low-drag one, our goal is to provide a trade-off between aerodynamics and acoustics. Thus Nash games are adopted to define a low-boom configuration close to aerodynamic optimality w.r.t. wave drag.
\end{abstract}

\section{Nomenclature}

$\begin{array}{ll}\text { BC } & \text { Boundary condition } \\ c_{D} & \text { Drag coefficient } \\ c_{L} & \text { Lift coefficient } \\ c_{L_{0}} & \text { Target lift coefficient } \\ \mathrm{DV} & \text { Design variable } \\ H & \text { Hessian matrix } \\ H^{\prime} & \text { Reduced Hessian matrix } \\ h & \text { Eigenvalue } \\ K & \text { Number of constraint functions }\end{array}$

*Ph. D. student, Applied Aerodynamics Department, Civil Aircraft Unit.

$\dagger$ Aeronautical Research Engineer, Applied Aerodynamics Department, Civil Aircraft Unit.

$\ddagger \mathrm{Ph}$. D. student, Opale Project-Team.

${ }^{\S}$ Research Director, Opale Project-Team, Head. 


$\begin{array}{ll}\text { LHS } & \text { Latin Hypercube Sampling } \\ n & \text { Number of design variables } \\ N & \text { Number of cost functions } \\ \text { MGDA } & \text { Multiple Gradient Descent Algorithm } \\ P & \text { Projection matrix } \\ \mathcal{S}^{\star} & \text { Pareto optimal set } \\ \text { SSBJ } & \text { Supersonic business jet } \\ u & \text { Descent direction } \\ \mathcal{U} & \text { Convex hull of gradients } \\ J & \text { Objective cost function } \\ \alpha & \text { Coefficient for linear combination of gradients } \\ \epsilon & \text { Continuation parameter } \\ \Lambda & \text { Eigenvalues matrix } \\ \Omega & \text { Split matrix } \\ \omega & \text { MGDA descent direction } \\ \rho & \text { Descent step length } \\ \theta & \text { Relaxation factor } \\ g & \text { Constraint function } \\ h_{Z} & \text { Flight altitude } \\ x & \text { Design variables set } \\ C F D & \text { Computational Fluid Dynamics } \\ A o A & \text { Angle of Attack }\end{array}$

\section{Introduction}

In shape design, optimization methods have acquired in the last two decades an increased maturity and are more currently used in the engineering design processes. When more than one discipline is considered, shape optimization techniques introduce a potentially significant advantage with respect to traditional engineering design strategies to provide a set of optimal solutions. In optimum-shape design, often the different physical phenomena are antagonistic. The models that describe each discipline are solved in domains that are identical or distinct but share a common geometrical boundary at which appropriate conditions are enforced. In this work, we refer to concurrent engineering problems, where global design variables common to all disciplines have to be optimized concurrently to account for antagonistic criteria.

Different methods are available in the literature to solve of a multi-objective problem, several surveys exist $^{1}{ }^{2}$ The simplest approach is the weighted sum method meant to minimize a single functional agglomerating all the criteria weighted by arbitrary penalty coefficients. This method can easily to be implemented and many single-objective optimization algorithms can be adopted. Nevertheless, the main drawback of this method is that it only provides one point that belongs to the targeted Pareto front for a given combination of weights. Another class of algorithms aim at the identification of non-dominated set of solutions. Among these algorithms, multi-objective algorithms based on evolutionary strategies have acquired a wide diffusion because of their ability to capture a set of distributed solutions on the Pareto front ${ }^{3}{ }^{4}$. For this reason most of the approaches (e.g. MOGA, ${ }^{5} \mathrm{PAES},{ }^{6}$ SPEA, ${ }^{7}$ NSGA-II ${ }^{8}$ ) to multi-objective problems are based on evolutionary strategies. ${ }^{9}$

The antagonism between the different objectives does not necessarily prevent the development of a primary phase of optimization by a cooperative algorithm, throughout which all objective-functions improve; a design-point on the Pareto set is thus found; then, a secondary phase of optimization by a competitive algorithm may be devised to generate new design-points along a path that is by construction tangent to the Pareto set. Practically speaking, our goal is thus to improve a secondary objective, without departing excessively from Pareto-optimality.

The paper is organized as follows: in the first part algorithms based on cooperation and competition strategies are introduced. Their main features and properties are described, and highlighted on analytical test cases. Both are combined in a cooperative-competitive strategy to explore the Pareto front. In the second part of the paper these approaches are adopted for the aero-acoustic shape optimization of a supersonic business jet configuration. Finally conclusions and new perspectives are given. 


\section{Cooperation and competition}

Cooperation and competition are two natural laws which regulate several aspect of nature, economy and social relations. As already made for genetic algorithm, ant-colony or simulated annealing, optimization algorithms usually take inspiration from natural laws. In this section competition and cooperation are exploited in two multi-objective approaches with complementary merits: a multiple gradient descent algorithm and a Nash game strategy. In order to introduce their main features, they are described and analyzed first on analytical test cases.

\section{II.A. Multiple gradient descent algorithm}

Our goal is to define a solution or a set of solutions that belong to the Pareto-optimal set $\mathcal{S}^{\star}$. The fundamental idea is to identify a descent direction from the given initial point $\mathbf{x}^{0}$ as a natural extension of the singleobjective steepest descent method. In the steepest descent method, the descent direction $\omega$ is defined as $-\nabla J(\mathbf{x})$, where $J$ is the cost function considered. A multiple gradient descent algorithm (MGDA) has been developed by Désidéri. ${ }^{12} \omega$ is defined in MGDA as the minimum norm element in the convex hull of the gradients: ${ }^{11}$

$$
\begin{gathered}
\omega=\operatorname{Argmin}_{u \in \overline{\mathcal{U}}}\|u\| \\
\text { where } \overline{\mathcal{U}}=\left\{u \in \mathbb{R}^{n} / u=\sum_{i=1}^{N} \alpha_{i} \nabla J_{i}\left(\mathbf{x}^{0}\right) ; \alpha_{i} \geq 0(\forall i) ; \sum_{i=1}^{N} \alpha_{i}=1\right\}
\end{gathered}
$$

A consequence of this definition is the following:

$$
\forall u \in \overline{\mathcal{U}}: \quad(u, \omega) \geq\|\omega\|^{2}
$$

and since this applies in particular to the gradients of the objective functions $\left(u=\nabla J_{i}(\mathbf{x}) / \forall i\right),-\omega$ is a descent direction common to all criteria. The descent iteration is written as:

$$
\mathbf{x}_{n e w}=\mathbf{x}_{\text {old }}-\rho \omega\left(\mathbf{x}_{\text {old }}\right)
$$

where $\rho$ is the step length (line-search). In multi-criterion optimization, it is not always easy to compute a step size that is satisfactory w.r.t. all criteria and ensuring a significant evolution toward the Pareto front. The step length is assumed to be the minimum step that decreases all the functional considered. Iterations are performed until the condition of Pareto stationarity condition is satisfied:

Definition II.1 (Pareto-stationarity) A point $\mathbf{x}$ is said to be Pareto-stationary if there exists a convex combination of the gradients, $\nabla J_{i}(\mathbf{x})$, equal to 0 :

$$
\exists \alpha \in \mathbb{R}^{N} \text { such that } \alpha_{i}>0(\forall i) ; \quad \sum_{i=1}^{N} \alpha_{i}=1 ; \quad \sum_{i=1}^{N} \alpha_{i} \nabla J_{i}(\mathbf{x})=0
$$

If $\mathbf{x}$ is a Pareto-optimal design-point in some open domain of the admissible domain assuming locally convex objective functions, then it satisfies the above Pareto-stationarity condition; furthermore, accumulation points of MGDA are Pareto-stationary. ${ }^{11}$ In Zerbinati et al. ${ }^{13}$ the ability of MGDA to identify the Pareto set was demonstrated after a comparison with the algorithm PAES.

Two main aspects have to be taken into account when complex shape optimization problems are analyzed: computational time and constraints handling. Disciplinary analysis are usually based on high fidelity models which are costly in terms of computational time. If finite difference evaluations of the gradients are required within the optimization process, the computational time tends to dramatically increase. To overcome this difficulty a meta-model assisted version of MGDA has been developed and applied to an engineering shape optimization problem by Zerbinati et al. ${ }^{14}$ It consists in a mixed-fidelity approach (see Fig. 1). An initial set of design points is generated using a sampling method in $\mathbb{R}^{N}$. Each configuration is evaluated using a high-fidelity model, then the evaluated points are used for the definition of surrogate models. The databases are also used to provide starting points to initiate the MGDA method, which is conducted until convergence using gradients that are calculated on the basis of the metamodels. Each converged point belongs to the Pareto set associated with the two-criterion problem related to the metamodels. The entire Pareto set is then 


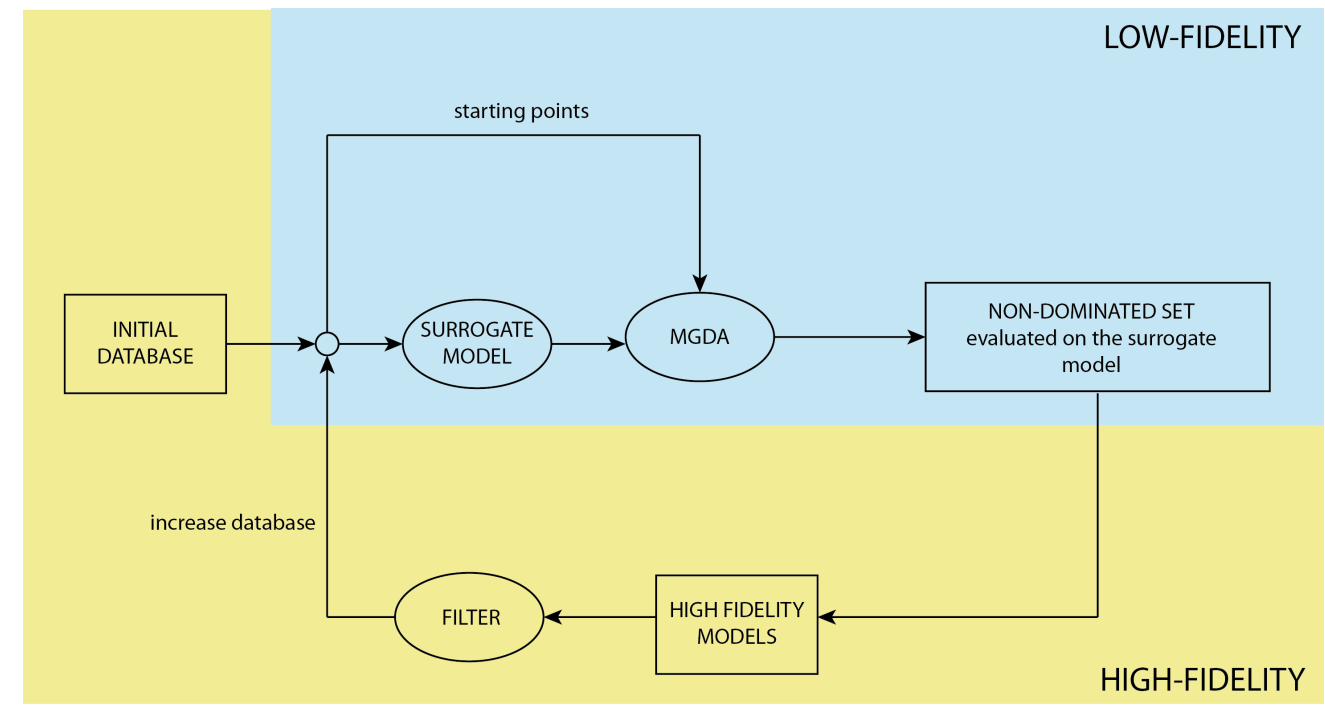

Figure 1. MGDA with surrogate model scheme. A surrogate model based on an initial database is trained. Then, MGDA is applied from each database point. Thus a non dominated set on the surrogate model is obtained. Unless a non dominated point obtained is too close to an initial database element, its exact performance is computed and used to increase the database.

re-evaluated by a flow computation, and added to the database. A filtering method is applied to remove a point if it is too close to an existing point. At completion of this database enrichment process, the metamodel is updated. Several mixed-fidelity iterations are required to converge to the real Pareto front but with a limited computational cost.

When a constrained problem is considered two approaches are possible:

- consider an equivalent unconstrained problem using penalty functions;

- project the descent direction in the direction tangent to the constraints surfaces taking eventually care of push-off factors ${ }^{15}$ in order to descend into a feasible region.

The first methodology is easy-to-use and does not require particular attention with the exception of the penalty function weights definition and will be later applied in the aero-acoustic shape optimization problem coupled with the meta-model assisted MGDA. The second approach is adopted in the following test case. Consider the multi-objective constrained analytical problem:

$$
\operatorname{Minimize}\left\{\begin{array}{l}
J_{1}(\mathbf{x})=\left(x_{1}-2\right)^{2}+\left(x_{2}-2\right)^{2}+2 \\
J_{2}(\mathbf{x})=9 x_{1}-\left(x_{2}-1\right)^{2}
\end{array}\right.
$$

subject to the following inequality constraints:

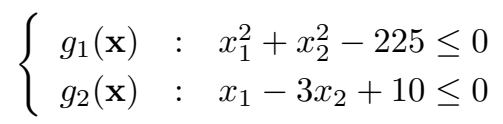

where $x_{i} \in[-20,20]$.

MGDA has been coupled with the algorithm CONMIN $^{15}(\mathrm{CM})$ to exploit its features when constrained problems are considered in a MGDA-CM version. The descent direction $\omega$ is provided by MGDA, the linesearch is performed on the two functions and the minimum step between the two is adopted. The constraints handling technique based on the method of feasible directions is performed by CONMIN. The convergence toward the Pareto front is ensured when the condition of Pareto stationarity is satisfied. Figure 2 shows the comparison between the Pareto front obtained using MGDA-CM starting from 200 initial design points selected using the LHS method, and the one obtained using NSGA-II ${ }^{8}$ with a 12 individuals population and 100 generations performed. The result shows that MGDA-CM is able to identify a well distributed non dominated set with a reduced computational cost. Three versions of MGDA have been described, in table 1 


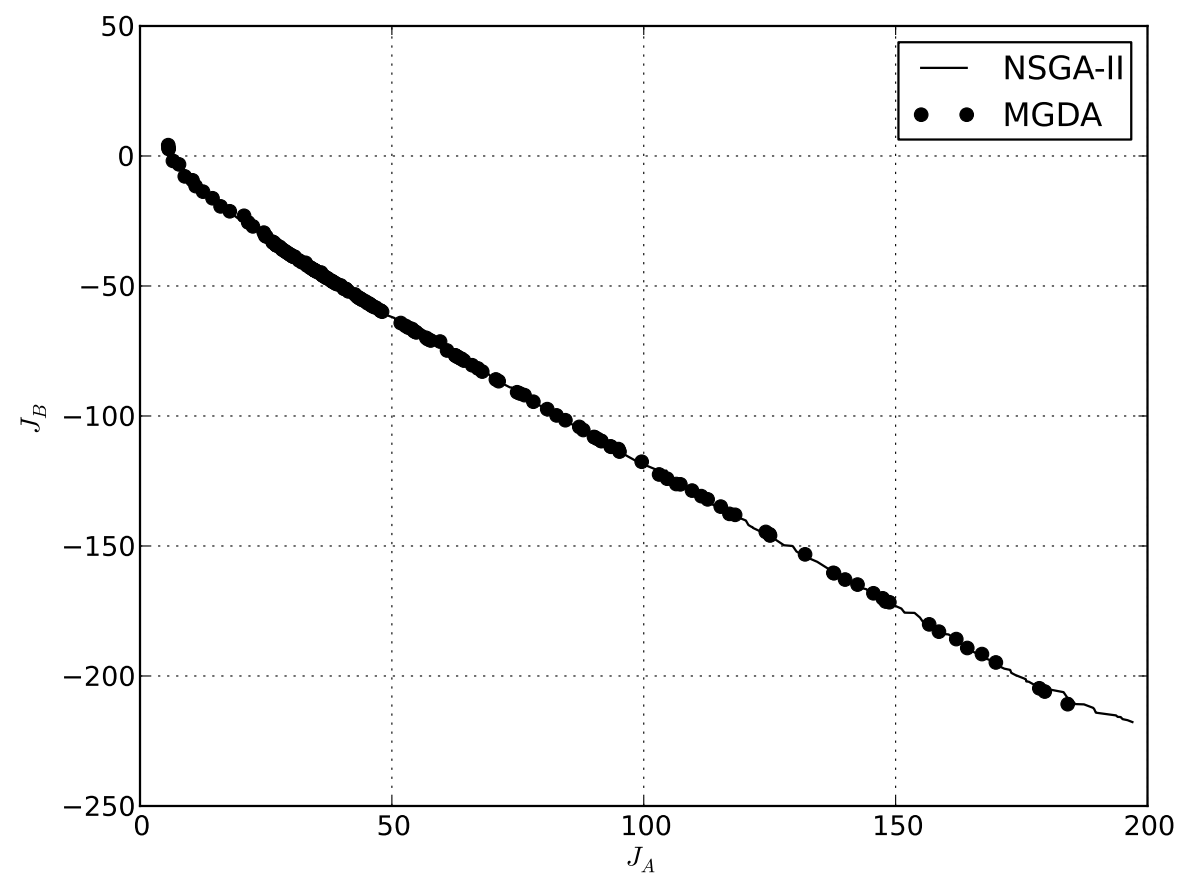

Figure 2. Pareto front obtained with MGDA-CM compared with the result obtained using NSGA-II.

\begin{tabular}{|cccc|}
\hline \hline Version & Model & Gradient evaluation & Constraints handling \\
\hline MGDA & high or low fidelity & model provided & penalty functions \\
MA-MGDA & mixed & low-fidelity model & penalty functions \\
MGDA-CM & high or low fidelity & model provided & method of feasible directions \\
\hline \hline
\end{tabular}

Table 1. Differences between MGDA versions.

main features of each of them are resumed.

MGDA exploiting cooperation is able to provide one or more solutions on the Pareto front. Once a solution is on the Pareto front one could decide to stop the optimization process or to introduce competition and hierarchy among disciplines performing a Nash game.

\section{II.B. Nash game and splitting of territories}

A game is a model that simulates the interaction between different agents, or players. Each player adopts a proper strategy to improve or optimize one's own "pay-off" function using partial or complete information about the strategies of the other players. Competitive games, also called non-cooperative games, have been introduced by Nash. ${ }^{16}$ Games in which a particular player, the leader, makes one own's decision first, and the other players, the followers, make their decision subsequently, are known as Stackelberg games; such games are not considered presently.

We are going to consider optimization problems involving two objective functions $J_{A}(x)$ and $J_{B}(x)$ to be minimized. By an appropriate change of variables (to be defined later) the design vector $\mathbf{x}$ is made a function of two sub-vectors $U$ and $V$ :

$$
\mathbf{x}=\mathbf{x}(U, V)
$$

where $\operatorname{dim} U+\operatorname{dim} V=\operatorname{dim} \mathbf{x}$. In this way $J_{A}(\mathbf{x})=J_{A}(U, V)$ and $J_{B}(\mathbf{x})=J_{B}(U, V)$. Then following Tang et al. ${ }^{10}$ and Désidéri, ${ }^{11}$ the subvectors $U$ and $V$ are used as strategies in a symmetrical Nash game to improve $J_{A}$ and $J_{B}$ respectively.

In a symmetrical Nash game, an equilibrium point is found when each player's set of action parameters 
realize a local optimum of one's own pay-off subject to the action parameters decided by the other players. For two players, this can be reformulated as follows:

Definition II.1 (Nash equilibrium) Consider a symmetrical Nash game with two players $A$ and $B$. The strategy pair $\bar{x}=(\bar{U}, \bar{V})$ represents the Nash equilibrium for the two players iff :

$$
\left\{\begin{array}{l}
\bar{U}=\operatorname{Argmin}_{U} J_{A}(U, \bar{V}) \\
\bar{V}=\operatorname{Argmin}_{V} J_{B}(\bar{U}, V)
\end{array}\right.
$$

Each player uses a subset of $\mathbf{x}$, respectively $U \in \mathbb{R}^{n-p}$ and $V \in \mathbb{R}^{p}$, to minimize their discipline functional. In our method, an $n \times n$ splitting matrix $\Omega_{H}$ is defined and the following change of variables is adopted:

$$
\mathbf{x}=\mathbf{x}_{A}^{\star}+\Omega_{H}\left(\begin{array}{l}
U \\
V
\end{array}\right)
$$

The definition of the splitting matrix becomes a crucial aspect in the optimization process. In general, the choice of the $\Omega_{H}$ matrix requires an a priori knowledge of the physics of the phenomenon. ${ }^{10}$ In Désidéri ${ }^{11,18}$ a method to define an adequate split of territories is proposed with a complete mathematical formulation. For this purpose we proceed as follows.

1. Identify the optimum solution associated with the single-objective problem related to the primary discipline:

$$
\begin{aligned}
& \mathbf{x}^{\star}=\operatorname{Argmin}_{A}(\mathbf{x}) \\
& \text { subject to } g(\mathbf{x})=0
\end{aligned}
$$

2. Assume that the approximations of the gradient $\nabla J_{A}\left(\mathbf{x}_{A}^{\star}\right)$, the Hessian matrix $H_{A}^{\star}=H_{A}\left(\mathbf{x}_{A}^{\star}\right.$ and the gradient of the constraints $\nabla g\left(\mathbf{x}^{\star}\right)$ are available. If it is not the case it is possible to compute an approximation adopting a surrogate model of discipline A and associated constraints. Considering a constrained problem, if $K \geq 1$ constraints are active, the Gram-Schmidt orthogonalization process is applied to the constraint gradients $\left\{\nabla g_{k}\right\}$. This results in a set of orthogonal unit vectors $\left\{\omega_{k}\right\}$, spanning the same subspace. Then, a projection matrix $P$ is defined as follows:

$$
P=I-\sum_{k=1}^{K}\left[\omega_{k}\right]\left[\omega_{k}\right]^{T}
$$

where $\left[\omega_{k}\right]$ denotes the $n \times 1$ column-vector, and the superscript ${ }^{T}$ stands for transposition. The projected Hessian is then written as follows:

$$
H_{A}^{\prime}=P H_{A}^{\star} P
$$

Applying a spectral decomposition:

$$
H_{A}^{\prime}=\Omega_{H} \Lambda_{H} \Omega_{H}^{T}
$$

where the split matrix is fully defined after the ordering of the eigenmodes is chosen such that the eigenvalues $\left\{h_{i}\right\}$ of the projected Hessian $H_{A}^{\prime}$ satisfy: $h_{i}=0$ for $i=1, \ldots, K$, and $h_{K+1} \geq h_{K+2} \geq$ $\cdots \geq h_{n}$. For an unconstrained problem the projection matrix $P$ is the identity matrix and the spectral decomposition is performed directly on $H_{A}^{\star}$.

If the objective of the optimization is to optimize two different disciplines where one is considered primary or fragile, the interest is to introduce the optimization of the second discipline as a small perturbation from the original optimum of the primary cost function $J_{A}^{\star}$. The definition (II.1) could be applied directly to a multi-objective problem, ${ }^{17}$ but a more interesting revisited formulation of the concurrent optimization problem proposed by Désidéri ${ }^{18}$ defines this new multi-objective problem as:

$$
\left\{\begin{array}{l}
\min _{U} J_{A}(U, V) \\
\text { subject to }
\end{array} \quad g=0\right.
$$

$$
\left\{\begin{array}{lr}
\min _{V} J_{A B}(U, V)= & \frac{J_{A}}{J_{A}^{\star}}+\epsilon\left(\theta \frac{J_{B}}{J_{B}^{\star}}-\frac{J_{A}}{J_{A}^{\star}}\right) \\
\text { subject to } & \text { no constraints }
\end{array}\right.
$$




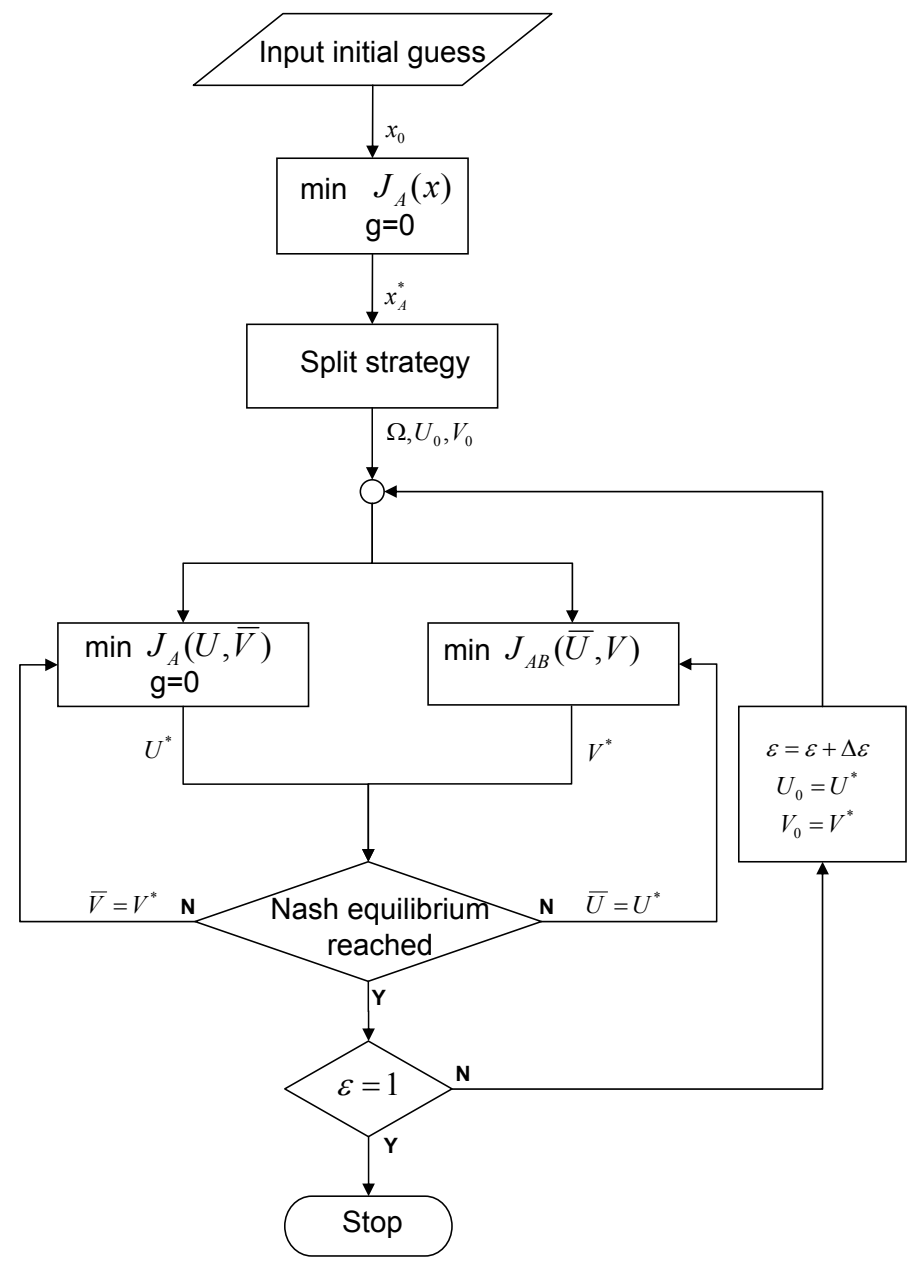

Figure 3. Flow-chart for the continuum Nash equilibrium in multi-objective optimization.

where $\epsilon$ is a continuation parameter that allows to smoothly introduce the antagonism between disciplines varying from 0 to 1 and $\theta$ is a relaxation factor usually set to 1 .

The complete procedure is sketched in figure 3 .

The main theoretical result (cf. Désidéri ${ }^{11}$ ) is that when $\epsilon=0$, the Nash equilibrium exists and it corresponds precisely to $\mathbf{x}_{A}^{\star}$ independently of the dimensions $n, p$ and $K$, thus guaranteeing the consistency of the Nash game for $\epsilon=0$ with the single-discipline optimization of discipline A alone. As $\epsilon$ increases, the continuum of Nash equilibrium is found $\overline{\mathbf{x}}=\overline{\mathbf{x}}_{\epsilon}=\mathbf{x}\left(\bar{U}_{\epsilon}, \bar{V}_{\epsilon}\right)$ The Nash equilibrium evolves smoothly from the optimum $J_{A}^{\star}$ solution of the problem for $\epsilon=0$ to the solution of the problem minimization of $J_{B}$ when $\epsilon$ progressively varies from 0 to 1 . Infinitesimal perturbations of the parameters lying on the secondary functional territory potentially cause the least possible degradation to the value of the primary functional so that $J_{A}\left(\overline{\mathbf{x}}_{\epsilon}\right)=J_{A}^{\star}+\mathcal{O}\left(\epsilon^{2}\right)$.

Now, consider as demonstrative test case the following multi-objective constrained problem:

$$
\operatorname{Minimize}\left\{\begin{array}{lll}
J_{A}(\mathbf{x})=\sum_{k=0}^{3} \frac{x_{k}^{2}}{3^{k}} & \text { subject to : } g=x_{0}^{4} x_{1}^{3} x_{2}^{2} x_{3}-96 \sqrt{3} \\
J_{B}(\mathbf{x})=\sum_{k=0}^{3} x_{k}^{2} & \text { subject to : } & \text { no constraints }
\end{array}\right.
$$


The minimum of the primary discipline $\mathrm{A}$ is $J_{A}^{\star}=3 . \overline{3}$ located at $\mathbf{x}^{\star}=(2 / \sqrt{3}, \sqrt{3}, \sqrt{6}, 3)$. and the corresponding $J_{B}\left(\mathbf{x}^{\star}\right)=19 . \overline{3}$

The Hessian, the gradient and the projected Hessian are respectively:

$$
H\left(\mathbf{x}^{\star}\right)=\left[\begin{array}{cccc}
2 & 0 & 0 & 0 \\
0 & 0 . \overline{6} & 0 & 0 \\
0 & 0 & 0 . \overline{2} & 0 \\
0 & 0 & 0 & 0.0741
\end{array}\right], \nabla g\left(\mathbf{x}^{\star}\right)=\left[\begin{array}{c}
576 \\
288 \\
135.77 \\
55.43
\end{array}\right], \quad H^{\prime}=\left[\begin{array}{cccc}
0.2186 & -0.3837 & -0.1012 & -0.0305 \\
-0.3837 & 0.7284 & 0.0689 & 0.0336 \\
-0.1012 & 0.0689 & 0.2735 & 0.0235 \\
-0.0305 & 0.0337 & 0.0235 & 0.0847
\end{array}\right]
$$

The splitting matrix $\Omega$ obtained after the spectral decomposition and the reordering is so defined as:

$$
\Omega=\left[\begin{array}{cccc}
0.8721 & 0.4748 & -0.1124 & -0.037 \\
0.4361 & -0.8637 & -0.2452 & -0.0607 \\
0.2056 & -0.1599 & 0.9582 & -0.1186 \\
0.0839 & -0.0543 & 0.0955 & 0.9904
\end{array}\right]
$$

A $(2+2)$ Nash game is performed using the Hessian based split. The optimization algorithms adopted for

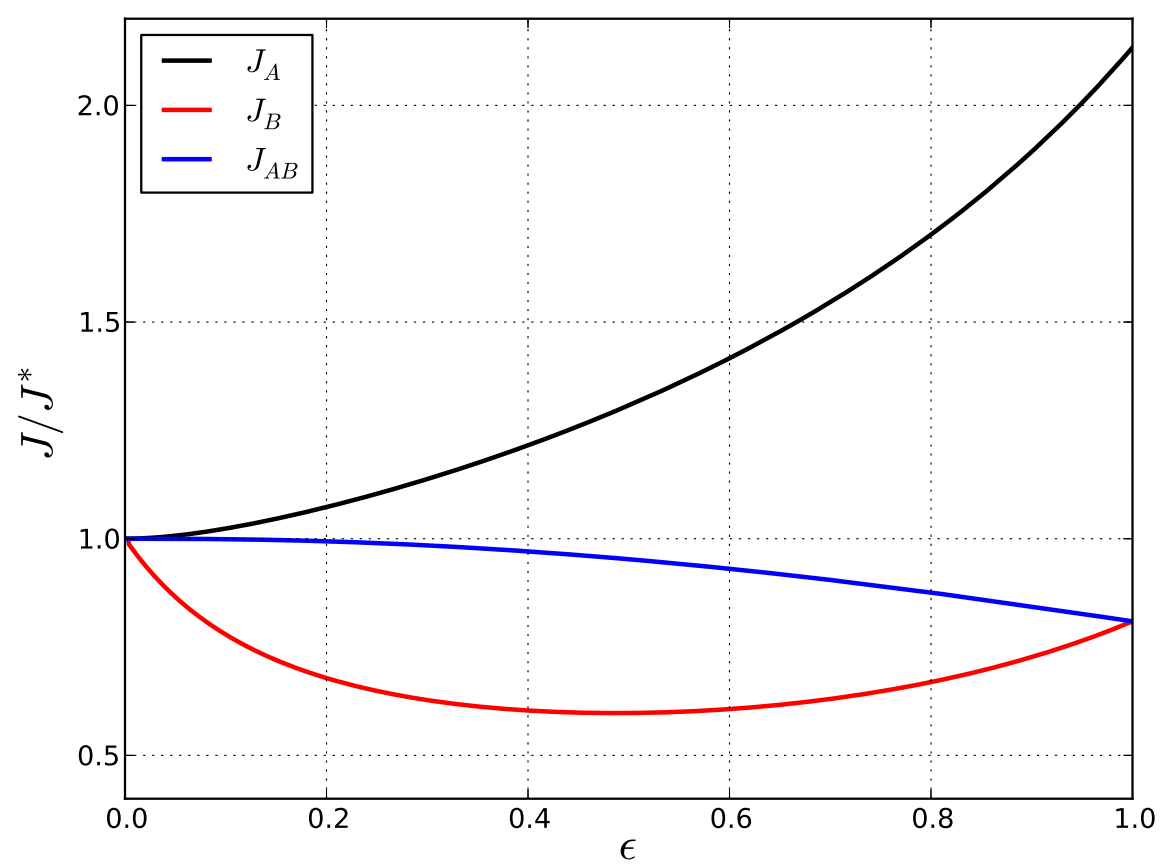

Figure 4. The continuum of Nash equilibrium points as $\epsilon$ varies. The $\epsilon$ step is taken equal to 0.001 , (2+2) Nash games are performed using the Hessian based split.

$J_{A}$ and $J_{B}$ are respectively Cobyla ${ }^{19}$ (Constrained problems by linear approximation of the constraints) and a Nelder-Mead simplex algorithm ${ }^{20}$ implemented in the Scipy.optimize ${ }^{21}$ Python library. The continuity parameter $\epsilon$ varies from 0 to 1 with a step of 0.001 and the relaxation parameter $\theta$ is set to 1 . For each $\epsilon$ we perform several iterations in order to satisfy the Nash equilibrium or reach the maximum number of iterations set to 20. Figure 4 shows the continuum of the Nash equilibrium as the continuity parameter $\epsilon$ varies. $J_{A}$ increases monotonically while $J_{B}$ is convex. The minimum of $J_{B}$ is achieved at $\epsilon=0.487$. Further increases of $\epsilon$ reveals to be counter-productive. The Nash game coupled with the split of territories based on the Hessian is able to reduce a second discipline without causing an excessive deterioration of the fragile discipline satisfying the constraints at each Nash equilibrium point. 


\section{II.C. Coupling cooperation and competition}

A natural extension consists in combining the cooperation and the competition phases for the exploration of the Pareto front. Consider two cost functions $J_{1}$ and $J_{2}$ that represent two antagonistic disciplines. The starting point of the process is the optimum of the first objective $J_{1}^{\star}$. This solution is a Pareto-stationary point that satisfies the definition II.1 for $\alpha=1$. Using the Hessian based split of territories it is possible to perform a Nash game moving in the tangent direction to the Pareto front at $x_{1}^{\star}$. The Nash game can be formulated as:

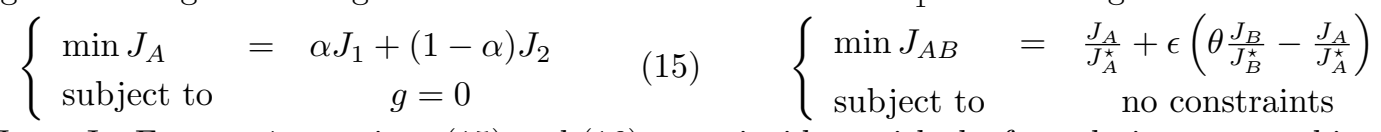

where $J_{B}=J_{2}$. For $\alpha=1$ equations (15) and (16) are coincident with the formulation proposed in equations (12) and (13). At the end of the competitive phase it exists at least one point that dominates the Nash equilibrium point and that belongs to the Pareto front. From a Nash equilibrium point, it is possible to perform a cooperative phase using MGDA and thus obtaining a Pareto stationary point that satisfy definition (II.1) for a different $\alpha$ with respect to the initial one.

A simple test case is here analyzed to introduce the procedure. The two criteria minimization problem is written as:

$$
\left\{\begin{array}{l}
J_{1}(\mathbf{x})=4 x^{2}+y^{2}+x y \\
J_{2}(\mathbf{x})=(x-1)^{2}+3(y-1)^{2}
\end{array}\right.
$$

The Pareto front can be described using the parametric form $J_{1}(\mathbf{x}(\alpha)), J_{2}(\mathbf{x}(\alpha))$ and evaluating the Pareto set of solutions $\mathcal{S}^{\star}$, that satisfy the Pareto-stationarity condition for different $\alpha$. The following steps are

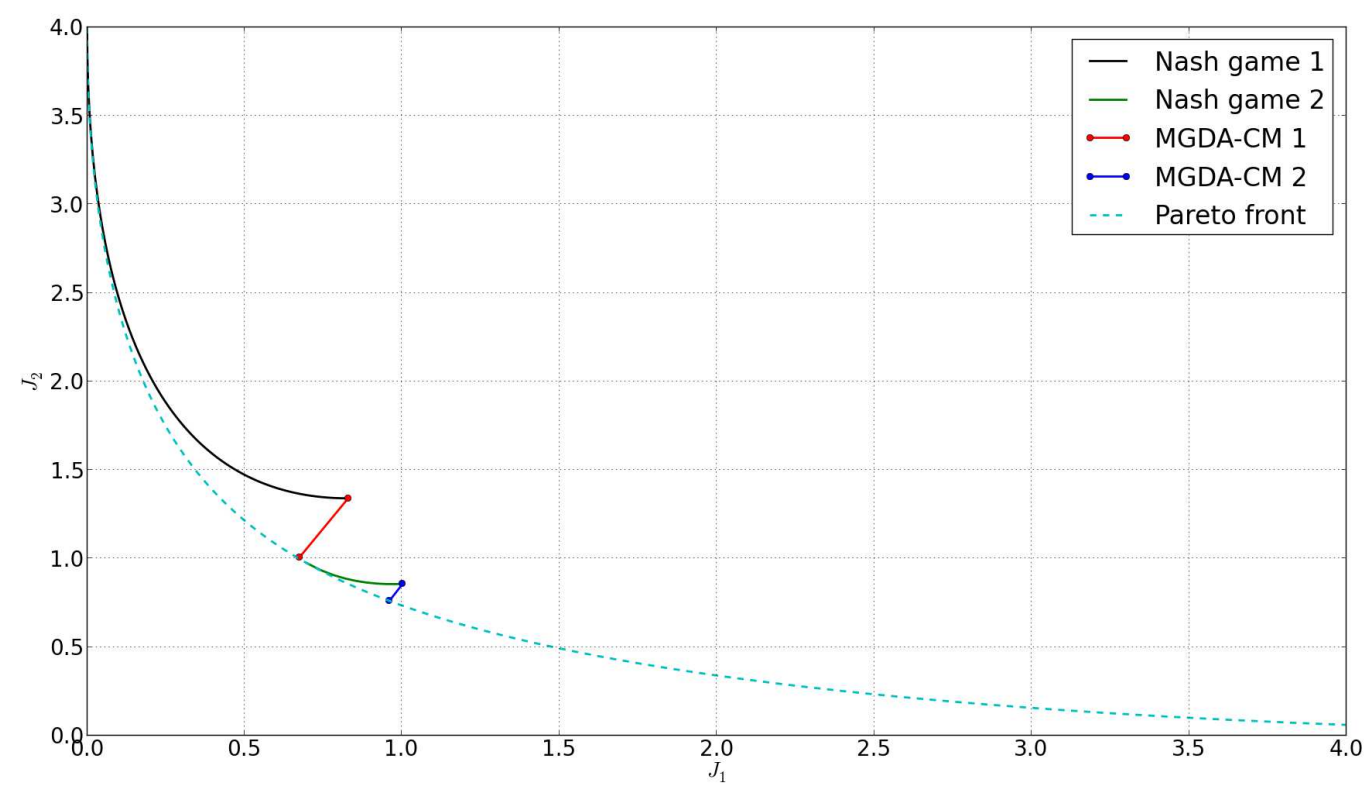

Figure 5. Coupling Nash games and MGDA iterations for the Pareto front exploration.

necessary for the cooperation/competition coupling applied to problem 17:

1. From the optimum of $J_{A}^{\star}(\alpha=1)$ located at $\mathbf{x}^{\star}=(0,0)$ the Hessian split of territories is evaluated and a Nash game $1+1$ is performed with $\alpha=1$;

2. From a Nash equilibrium point $\mathbf{x}_{n}$ a cooperation phase using MGDA is performed to obtain a solution $\mathbf{x}_{s}$ that belongs to the Pareto front;

3. A new $\alpha$ is evaluated in order to satisfy the Pareto-stationarity condition at $\mathbf{x}_{s}$;

4. A new split matrix is evaluated using the Hessian of the function $J_{A}(\alpha)$ where $\alpha$ has been evaluated at the previous point of the process; 
5. Points from 2 to 4 can be repeated to explore other regions of the Pareto front.

The procedure, repeated two times on problem (17), is shown in Figure 14.

\section{Aero-acoustic shape optimization of a wing-body supersonic configuration}

The optimization strategies described in the previous section are well suited for aeronautical shape design problems. Such design problems are by nature multi-disciplinary, where several disciplines interact for the definition of the final configuration. In the case of the development of a supersonic business jet, aerodynamics and acoustics represent the two antagonist par excellence. A body flying at supersonic speed, contrary to subsonic aircrafts, raises the problem of sonic boom in addition to aerodynamic performance. Pressure perturbations created at the flight altitude reach the ground, and are perceived by the human beings as an impulsive (the duration is around $100 \mathrm{~ms}$ ), low-frequency (the main part of the frequency spectrum is in the infrasonic or low-audible range) signal. ${ }^{22}$ Several studies, conducted recently ${ }^{23}$ and in the past ${ }^{24},{ }^{25}$ show that it is required to reduce the sonic boom annoyance in order to allow overland supersonic flight. This restriction represents actually the main limitation for the development of the supersonic civil transportation.

One of the most adopted approaches is to shape the configuration in order to reduce the ground pressure signature impact. The classical approach is based on the first order correction of the supersonic linearized theory proposed by Whitham. ${ }^{26}$ The Whitham correction is at the base of the development of the shaped sonic boom theory developed in the works of Jones, ${ }^{27}$ Petty, ${ }^{28}$ George,${ }^{29}$ George and Seebass, ${ }^{30}$ Darden, ${ }^{31}$ Rallabhandi, ${ }^{32}$ Plotkin $^{33}$ and culminates in the in-flight validations in the context of the DARPA project ${ }^{34}$ and more recently in the project D-SEND\#1. ${ }^{35}$ This method is a valuable tool in pre-design phases, but has a major drawback since it is not able to define a trade-off between two disciplines, typically aerodynamics and acoustics.

To overcome the limitation of the linearized theory ${ }^{36}$ and exploit CFD computations, nowadays shape optimization is coupled with the solution of Euler or Navier-Stokes equations. The problem of introducing a trade-off between disciplines still persists especially for non academic fully three dimensional configurations. $\mathrm{Choi}^{37}$ has been one of the first to consider a multi-objective aero-acoustic shape optimization of a SSBJ configuration using low-fidelity surrogate based models. The use of high-fidelity models for the multiobjective optimization is still nowadays computationally expensive. A first attempt in this sense is presented in Minelli et al. ${ }^{38} \mathrm{~A}$ multi-objective genetic algorithm coupled with high-fidelity models, has been used to optimize the aerodynamic performance and reduce the sonic boom of a wing-body supersonic configuration.

Concerning numerical prediction of sonic boom, the most accurate and efficient method available today is based on three layer decomposition method. ${ }^{39}$ The CFD computation is performed in the near-field and a multipole decomposition for near to far-field matching is performed. ${ }^{40}$ The acoustic perturbation is then propagated to the ground through a stratified atmosphere with an acoustic code (e.g non-linear acoustic ray-tracing).

In this work, the Onera elsA CFD code ${ }^{41}$ is associated to the evaluation of the aerodynamic near-field. elsA is able to solve internal and external aerodynamics problem performing Euler, RANS, URANS, DES, or LES simulations for mono-species perfect gas hypothesis. The flow equations are solved by a cell centered finite-volume method, and the flow regimes range from the low subsonic to the high supersonic regime. Inviscid Euler equations are used to describe the flow in the near-field. This choice is essentially due to two main reasons:

- The pressure signature in the near-field is predicted with sufficient accuracy. Generally Euler solutions shows sharper and more exaggerated peaks and dips in the pressure signature with respect to NavierStokes solutions ${ }^{42}$

- The CFD computation will be a part of an optimization process that requires several evaluation. Euler computations require less time to converge with respect to N-S computations, thus resulting in a limited computational cost.

The Roe flux ${ }^{43}$ with the Harten entropy correction ${ }^{44}$ is used. The Van Albada flux limiter ${ }^{45}$ is used to preclude oscillations due to shocks and discontinuities by limiting the values of the spatial derivatives.

Concerning the mesh adopted, figure 6 shows the grid topology. Structured CFD grids on the half configuration were used in the calculations. Four blocks model the flow domain that extends from the configuration up to $R / L=4$. The domain is a half cylinder with a conical cap and base that consists in 
$6 \cdot 10^{6}$ nodes. As outlined in ${ }^{42}$ the pressure signature is very sensitive to the mesh. To reduce numerical dissipation a Mach aligned grid is adopted, where grid lines are parallel to Mach waves direction.

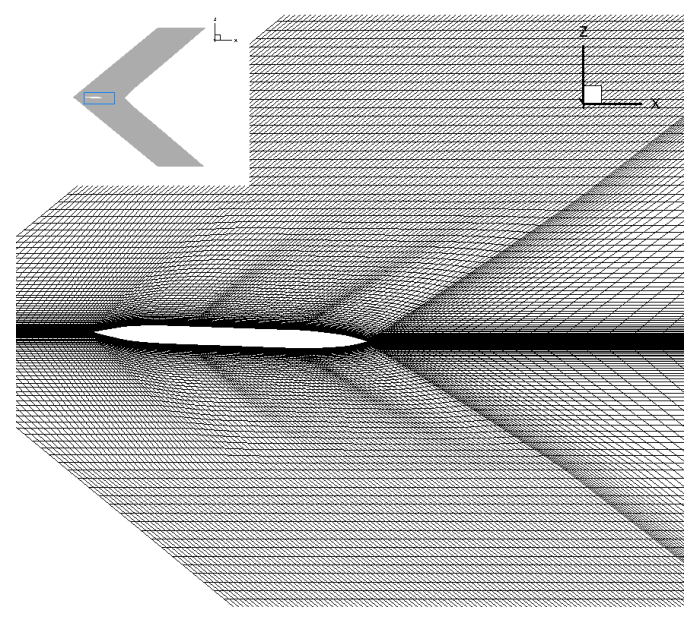

(a) Symmetry plane mesh

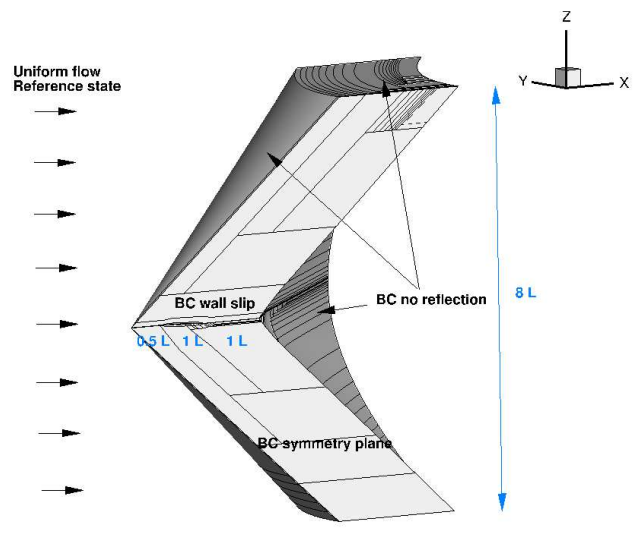

(b) Boundary conditions

Figure 6. Symmetry plane mesh and domain boundary conditions adopted for the elsA computations.

Non-reflection boundary conditions (BCs) were used at the inflow (left) boundary at the outflow (right) boundary and at far-field. A symmetry plane BC was used to model the axis of symmetry to compute only half configuration. Inviscid wall BCs were used to model the fuselage and the wing. Uniform free-stream flow conditions were used as initial conditions throughout the computational domain.

The near-field pressure is used to feed the matching phase. The pressure near field is interpolated on a cylinder aligned with the flow direction that surrounds the aircraft and used as input for the acoustic code TRAPS. ${ }^{46}$ No wind gradient and no turbulence are considered during signal propagation.

\section{III.A. Cooperation}

The objective of this section is to identify a Pareto set of solutions resulting from the trade-off of drag and sonic boom. The MA-MGDA algorithm is adopted to optimize the shape of a SSBJ wing-body configuration at the design condition $M_{\infty}=1.6, A o A=2 \mathrm{deg}$ and flight altitude $h_{Z}=18,000 \mathrm{~m}$. The aero-acoustic multi-objective problem can be stated as follows:

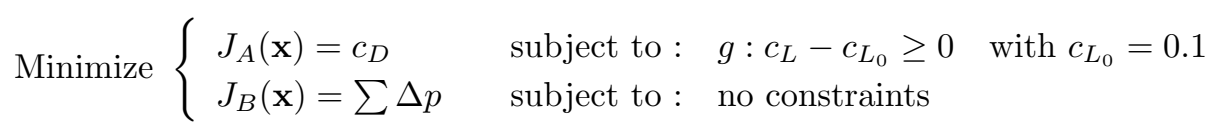

where $\mathbf{x} \in \mathbb{R}^{10}$ represents the vector of design variables. The objective cost function considered for aerodynamics is the drag coefficient with a constraint on lift, while the acoustic cost function is defined as the sum of the shocks overpressure of the under-track ground signature.

The geometrical variables that we want to modify represent different geometrical entities (lengths, angles and relative distances). Instead of their direct use in the optimization problem, the design set $\mathbf{x}$ is related to the geometrical set $\mathbf{g}$ as:

$$
g_{i}=\bar{g}_{i}\left(1+x_{i} \delta_{i}\right) \quad \forall i>1
$$

where $\bar{g}_{i}$ represents the geometrical variable of the reference configuration and $\delta_{i}$ is the maximum percentage modification allowed to each variable. An exception is made for the nose deflection variable as $\overline{g_{1}}$ is equal to 0 , the following relation is adopted: $g_{1}=0.5 x_{1}$. The design variables set $\mathbf{x}$ is dimensionless and can assume values between -1 and 1 . Table 3 shows the geometrical variables and the allowed modifications, while parameterization is shown in figure 7 .

The initial Kriging surrogate model has been defined using an LHS database of 100 points. Six MAMGDA complete iterations are performed, meaning MGDA convergence on the Kriging model, the evaluation of the Pareto set of solutions with high-fidelity models and Kriging model update. The MA-MGDA algorithm is able to identify the Pareto front of the high fidelity model with only 166 function evaluations. The NSGAII algorithm applied on the high-fidelity model is used as comparison in order to evaluate the quality of 


\begin{tabular}{|cccc|}
\hline \hline Variable & Specification & $\bar{g}_{i}$ & $\delta_{i}$ \\
\hline DV1 & nose deflection [deg] & 0 & - \\
DV2 & x-coordinate nose section [m] & 4 & $2 \%$ \\
DV3 & radius nose section [m] & 0.8 & $10 \%$ \\
DV4 & radius cabin section [m] & 1.015 & $10 \%$ \\
DV5 & x-coordinate rear section [m] & 28 & $2 \%$ \\
DV6 & radius rear section [m] & 0.535 & $10 \%$ \\
DV7 & dihedral angle [deg] & 3 & $100 \%$ \\
DV8 & relative wing position [adim] & 0.367 & $10 \%$ \\
DV9 & inner wing sweep angle [deg] & 65 & $10 \%$ \\
DV10 & outer wing sweep angle [deg] & 56 & $10 \%$ \\
\hline \hline
\end{tabular}

Table 2. Design variables set definition.

the solution evaluated using MA-MGDA. The comparison is performed at the same number of high-fidelity function evaluation calls, thus ensuring an identical computational cost. Results (see Figure 8) clearly show that the Pareto front evaluated using MA-MGDA completely dominates the solution obtained with NSGA-II. This identifies the efficiency of MA-MGDA, as it is able to converge more efficiently to the real Pareto front and is suited for computationally expensive problems. The diversity of the solutions is comparable between the two algorithms.

Three different configurations (see Table 3) that belong to the Pareto front (Figure 8) have been retained and compared in Figures 9 and 10.

\begin{tabular}{|ccc|}
\hline \hline Configuration & $J_{1}$ & $J_{2}$ \\
\hline Baseline & $89.01 \mathrm{dc}$ & $60.28 \mathrm{~Pa}$ \\
A & $71.72 \mathrm{dc}$ & $57.70 \mathrm{~Pa}$ \\
B & $73.98 \mathrm{dc}$ & $52.94 \mathrm{~Pa}$ \\
C & $83.14 \mathrm{dc}$ & $49.51 \mathrm{~Pa}$ \\
\hline
\end{tabular}

Table 3. Selected configurations that belong to the Pareto front.

The low drag configuration (A) and the trade-off configuration (B) show minor modifications on the fuselage geometry. The modifications impact mainly the wing plan-form. Configuration (B) shows a reduction of the dihedral angle with respect to the configuration $\mathrm{A}$ that is beneficial for the rear shock of the ground signature (see Figure 9(b)) with limited deterioration on the aerodynamic performance. In addition the dihedral angle actS modifying the acoustic footprint at ground, in particular the duration of the signature. The low boom configuration (C) shows a strong downward deflection of the nose. As a consequence the flow-field that reaches the wing leading edge is modified. The expansion before the wing shock is reduced and determines a reduction of the following shock. The front shock is almost not modified in any of the selected geometries. For the parameterization considered, the algorithm has identified the wing and the rear shock reductions as the most promising regions to improve the functions of interest.

Looking at the pressure distribution just below the aircraft in Figure 9(a) it is possible to note that the design variables are able to act and shape significantly all the shocks and expansion waves. In particular all the configurations show a split of the rear shock due to the combined modification of the wing plan-form and rear fuselage. The initial peak in the near field $p / p_{0}$ is reduced of nearly $20 \%$ with respect to the initial configuration, but this does not impact the ground level front shock pressure rise. In contrast the parameterization of the wing and of the rear part of the fuselage is able to produce modifications in the middle part of the near field signature. This corresponds to significant modifications on the ground signature pattern. As the sonic boom performance is improved, the pressure expansion just before the first maximum peak in the near-field signature is reduced, while the wing shock is increased in amplitude. The consequence at ground is a reduced amplitude of the second shock amplitude (from A to C). More design variables in the nose region should provide a better description of the shape, and thus improving the ability of the algorithm to reduce the front shock amplitude. 

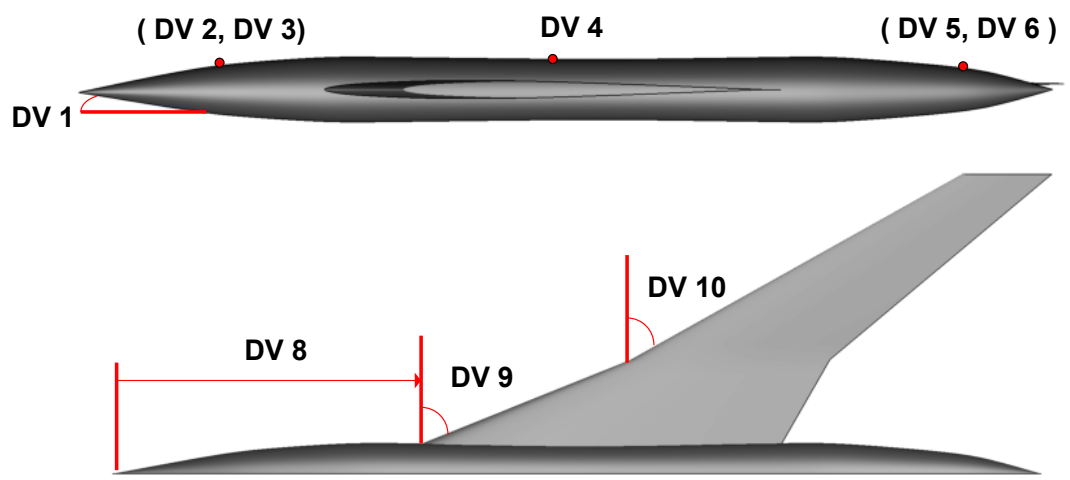

Figure 7. Wing-body parameterization.

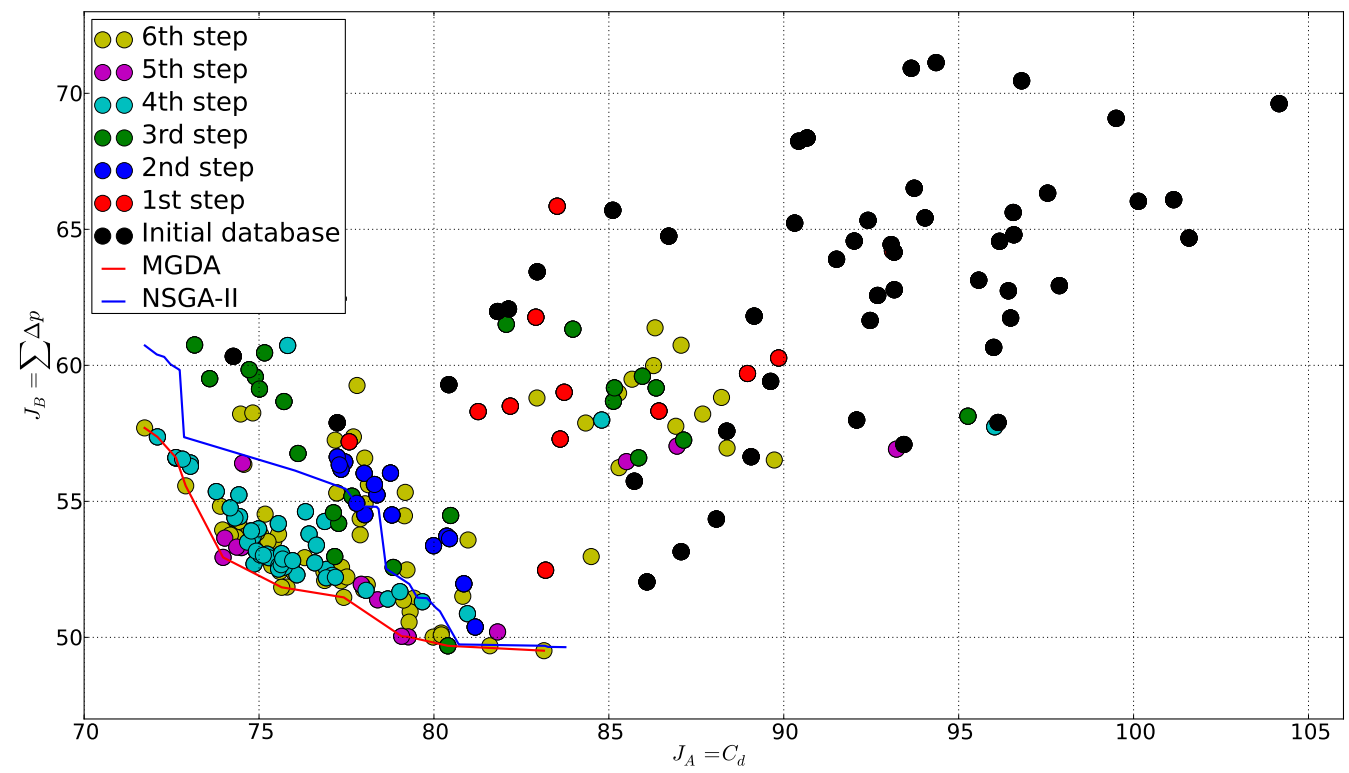

Figure 8. Convergence history at fixed maximum high fidelity function evaluation calls.

\section{III.B. Nash games applied to the aero-acoustic problem}

The same problem can be tackled from a competitive game point of view. Instead of a family of configurations that belong to the Pareto set, the result of the Nash game is a single configuration. The choice is to consider aerodynamics as preponderant and progressively introduce the sonic boom performance. The resulting configuration should show minimized sonic boom while preserving minimal aerodynamic performance degradation. 


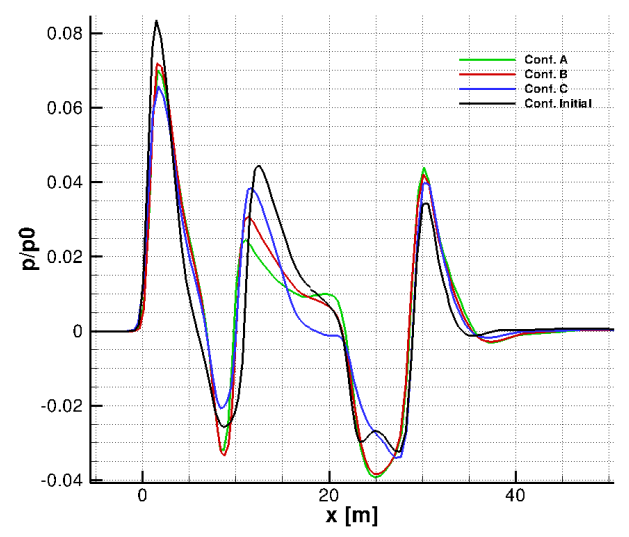

(a) Pressure signature $(p / p 0)$ extracted under-track at $\mathrm{z}=h_{Z}-10 \mathrm{~m}$

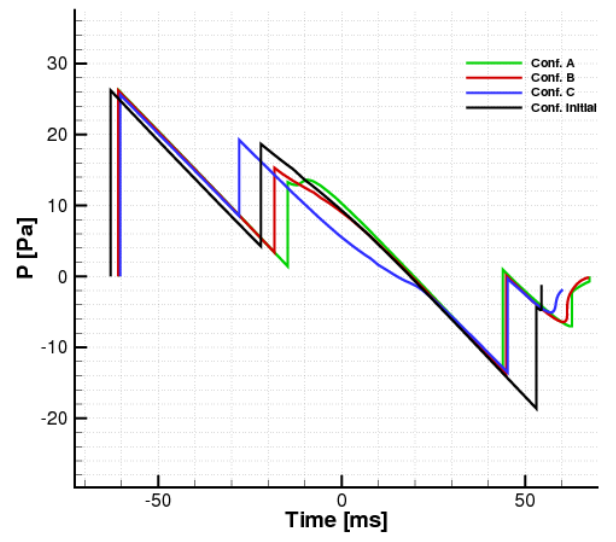

(b) Under-track ground signature

Figure 9. Near-field and ground pressure signal for different configurations on the Pareto front.

Concerning the model adopted, a mixed approach where the Nash game iterations are performed on a surrogate model and are finally evaluated using the high-fidelity CFD based model is adopted. This algorithm has been chosen for the following reasons:

- the Hessian based split of territories requires the evaluation of the Hessian at the optimum point of the primary discipline $H_{A}^{\star}$ and the gradient of the constraints $\nabla g_{A}^{\star}$. A surrogate model can provide these values without any additional function evaluations. Furthermore Hessian and gradient accuracy problems related to finite differences error are completely avoided;

- Nash game iterations requires many function evaluations for local disciplinary minimization of the two cost functional, that have to be repeated for several Nash game equilibrium iterations. In addition, if the continuum of the Nash equilibrium is the goal of the analysis, the process has to be repeated several times at each continuity iteration.

As a main difference with the approach adopted for the Metamodel Assisted MGDA, the surrogate model adopted cannot be updated along the iterations. In addition the surrogate model has to be accurate enough to predict the Hessian of the aerodynamic discipline. For this reason the construction of the surrogate models for each discipline requires particular care. Thus, the number of design variables considered has been reduced to 5 : nose deflection $\left(x_{1}\right)$, nose radius $\left(x_{2}\right)$, cabin radius $\left(x_{3}\right)$, wing position along the fuselage $\left(x_{4}\right)$, dihedral angle $\left(x_{5}\right)$. Design variables $\mathbf{x}$ are related to geometrical variables as in equation (19). Table 4 shows geometrical variables and the variation range allowed. Concerning nose deflection, the following

\begin{tabular}{|cccc|}
\hline \hline Variable & Specification & $\bar{g}_{i}$ & $\delta_{i}$ \\
\hline DV1 & nose deflection [deg] & 0 & - \\
DV2 & radius nose section [m] & 0.8 & $20 \%$ \\
DV3 & radius cabin section [m] & 1.015 & $15 \%$ \\
DV4 & relative wing position [adim] & 0.367 & $20 \%$ \\
DV5 & dihedral angle [deg] & 3 & $100 \%$ \\
\hline \hline
\end{tabular}

Table 4. Design variables set definition.

relationship is adopted: $g_{1}=0.7 x_{1}$.

A first kriging model for each discipline and for the constraint is created using a LHS database made up of 40 individuals. Five improvement iterations are performed to introduce points where the kriging model expected improvement is maximum. This procedure determines final databases that consist in 45 points. Cross-validation has been used to validate the final low-fidelity models. To evaluate the Nash equilibrium continuum the following steps are performed: 
A
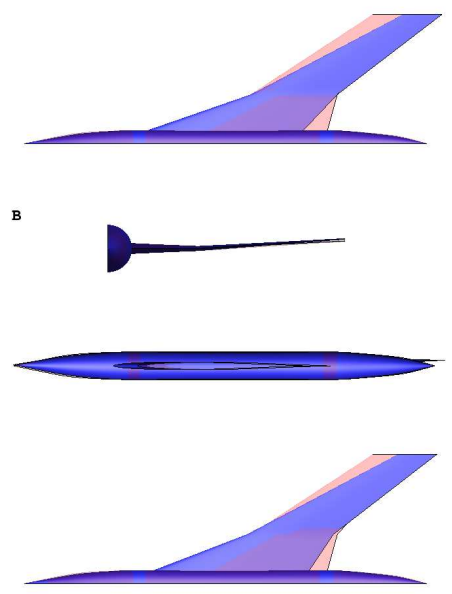

c
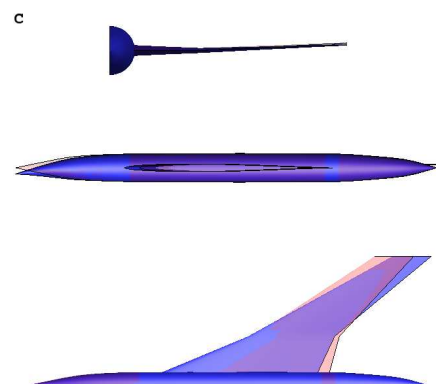
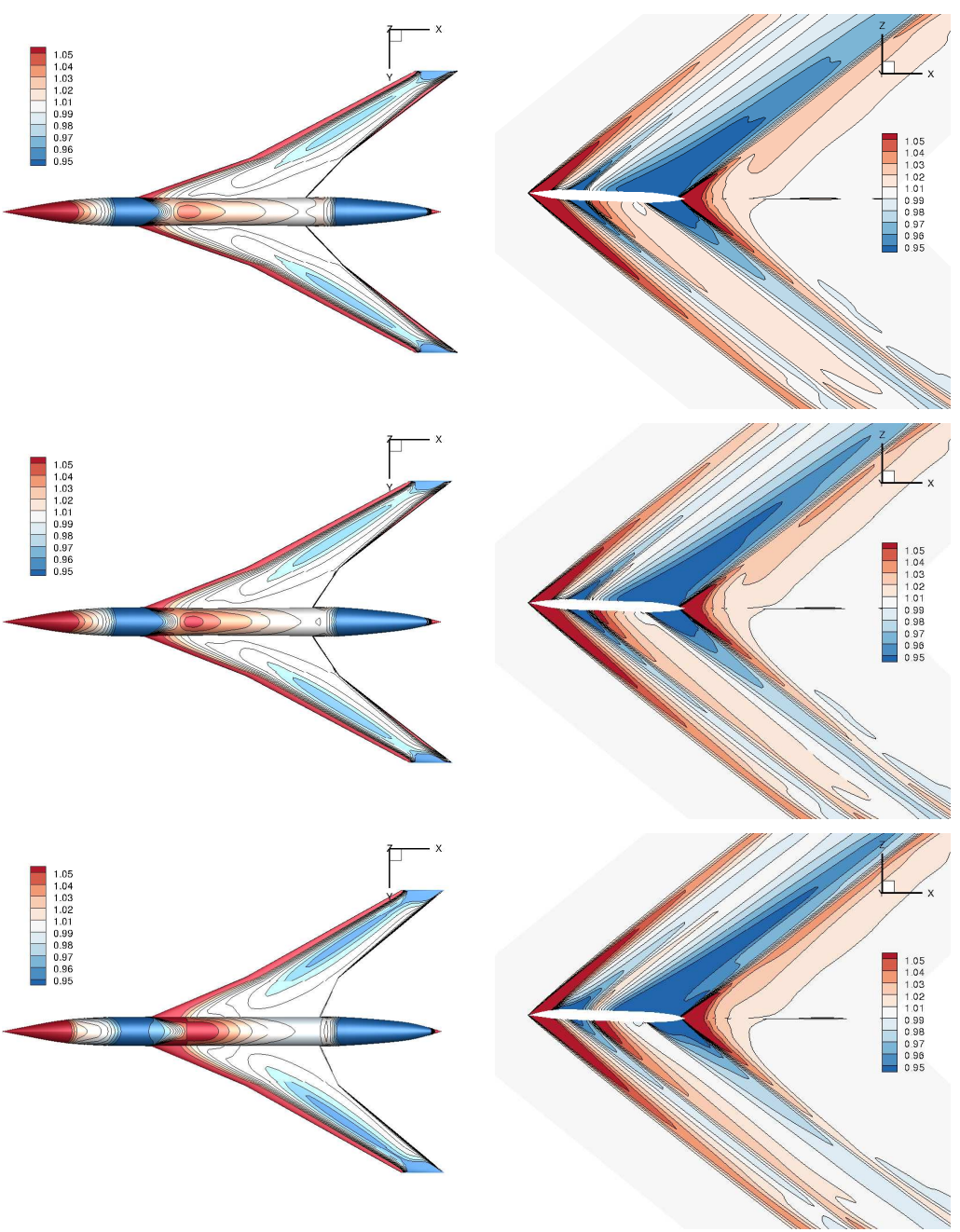

Figure 10. Geometry configuration, bottom skin pressure $\left(p / p_{0}\right)$ and symmetry plane pressure $\left(p / p_{0}\right)$ in near field for configuration $\mathrm{A}, \mathrm{B}$ and $\mathrm{C}$ on the Pareto front (In red the initial geometry).

- The minimization of the drag coefficient with constraint on lift $g: c_{l}-0.1 \geq 0$ is obtained using the algorithm CMA-ES. ${ }^{47}$ After less than 1200 iterations the optimum is found at $J_{A}^{\star}=81.458 \mathrm{~Pa}$ with $g=0.002$. The corresponding design vector is $\mathbf{x}=[-0.558,-0.836,-0.506,-0.434,-1]$;

- The Hessian-based split matrix $\Omega$ is evaluated using the procedure defined in section II.B. A split $2+3$ is adopted, where 2 variables are assigned to aerodynamics and the remaining to acoustics:

$$
\Omega=\left[\begin{array}{ccccc}
-0.1967 & -0.1839 & 0.0944 & -0.8251 & -0.4876 \\
-0.1257 & -0.4079 & -0.9042 & 0.0107 & 0.0114 \\
-0.6606 & -0.5744 & 0.3548 & 0.3282 & -0.0037 \\
-0.7055 & 0.6776 & -0.2077 & -0.0095 & 0.0049 \\
0.1070 & 0.1036 & -0.0672 & 0.4596 & -0.8730
\end{array}\right]
$$

- Nash game iterations are performed using as optimizer $\mathrm{SQP}^{48}$ for $J_{A}$ and a Nelder-Mead simplex algorithm for $J_{A B}$. Each single optimization is performed until convergence;

- Concerning continuity iterations, a step of 0.05 is considered for $\epsilon$.

Figure 11 shows the history of the continuum of the Nash equilibria. For $\epsilon=0$ all the three curves initiate at the value $1 . J_{A}$ and $J_{B}$ have zero slope due to the problem formulation defined in (13) which is consistent 


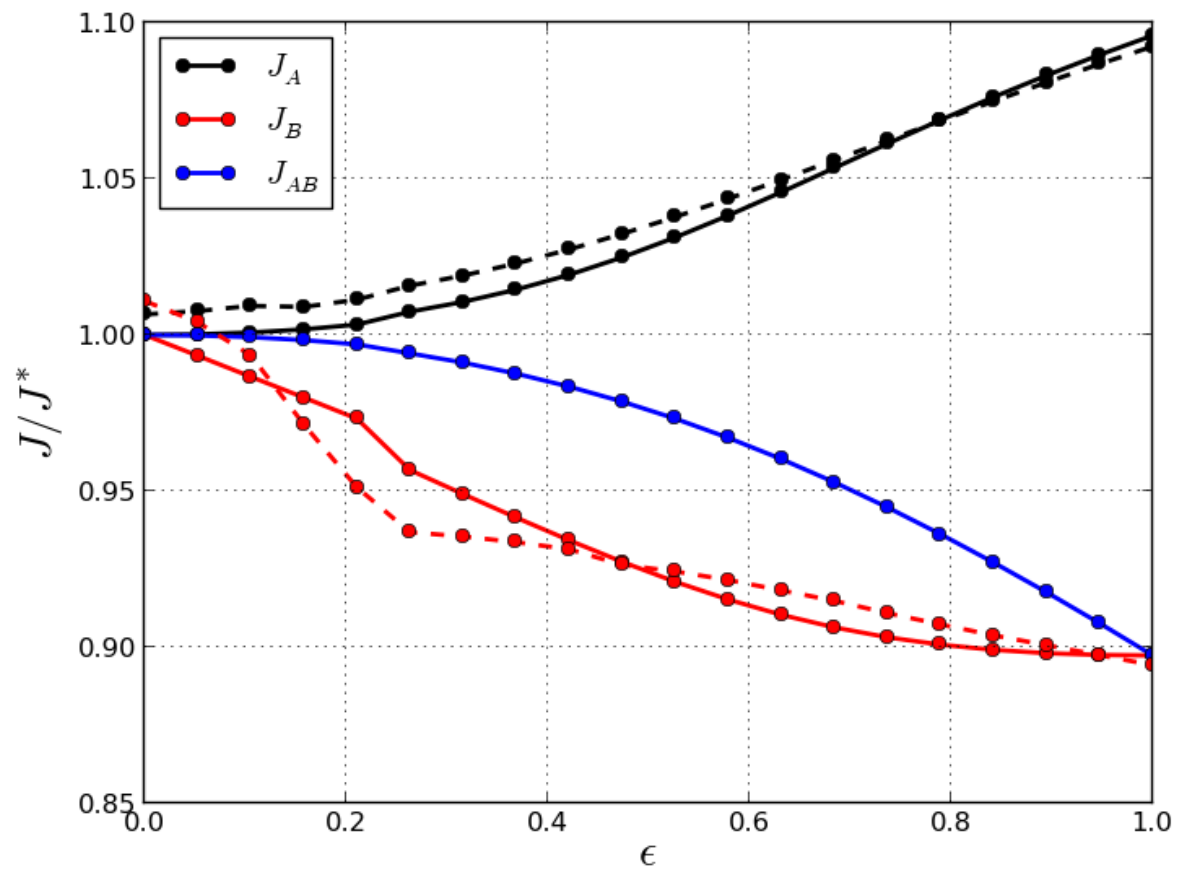

Figure 11. Continuum of the Nash equilibrium. Continuous lines represent evaluations using surrogate models, while dotted lines represents evaluations using high-fidelity models.

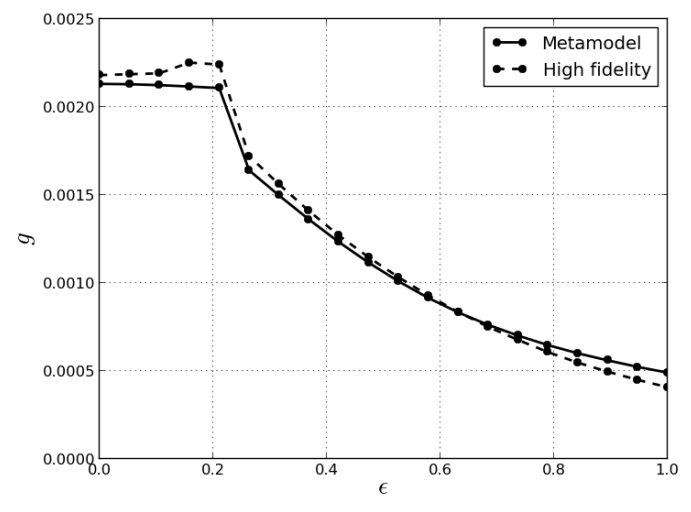

(a) Constraints function

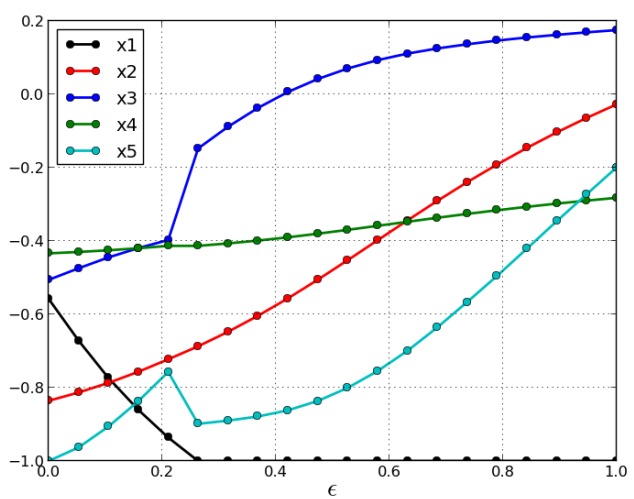

(b) Variables

Figure 12. Evolution of constraint function and variables during Nash game iterations.

with the original optimum. As $\epsilon$ increases drag degrades monotonically, while $J_{A B}$ and $J_{B}$ diminish. During the first iterations, the nose is deflected downward $\left(x_{1}\right)$ (see Figure $12(\mathrm{~b})$ ). At $\epsilon$ close to $0.25, x_{1}$ reaches its low-bound constraint. A new equilibrium point is obtained at the next iteration increasing the cabin radius $\left(x_{3}\right)$ and keeping the wing closer to the nose $\left(x_{5}\right)$. This new configuration has as consequence the increase of decreasing rate of $J_{B}$ and $g$. The low drag configuration has a dihedral angle $\left(x_{5}\right)$ equal to zero while as shown in Figure 15(c) for values of $\epsilon \geq 0.5$ the angle is almost equal to 3 degrees. The constraint function $g$ is satisfied along the iterations (see Figure 12(a)), but reducing the margin from the constraints activation when we are moving away from the aerodynamic optimum $\mathbf{x}_{A}^{\star}$.

The equilibrium points evaluated using the Kriging model are re-evaluated using the high fidelity models. The new curves in dotted lines in Figures (11) and (12(a)) are in agreement with the results obtained using the low-fidelity model. The accurate definition of the Kriging model has permitted to obtain the high 
correlation between low and high-fidelity model. From a design point of view solutions at point $\epsilon=0.25$ is the most interesting. In fact considering the high-fidelity prediction drag is degraded by only $1 \%$, while the acoustic function is reduced by more than $7 \%$.

In order to analyze the trade-offs an adequate visualization tool is required. Self-Organizing Maps $(\mathrm{SOM})^{49}$ are adopted to produce two-dimensional maps using neural networks. SOM preserves the topological properties of the input space aiming at analyze the different configurations obtained. The unified distance matrix (U-matrix) is a representation of a self-organizing map where the Euclidean distance between different neuron is depicted. Cluster regions that have the same code color mean neurons with similar topological properties. The U-matrix for the aero-acoustic problem in Figure 13(a) shows four cluster regions that are labeled respectively: low-boom (LB), high antagonism (HA), low antagonism (LA) and low drag (LD).
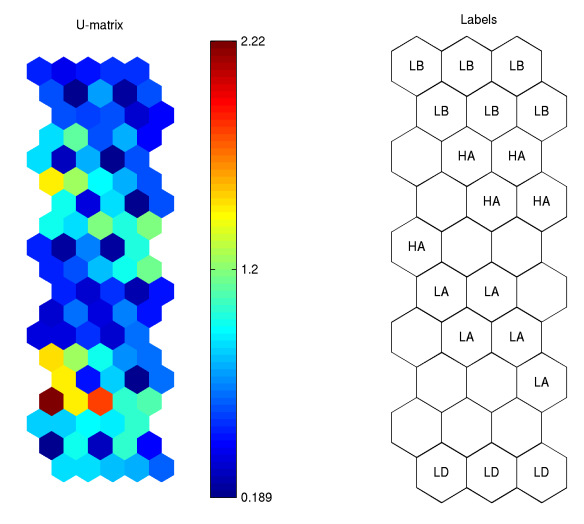

(a) U matrix SOM.
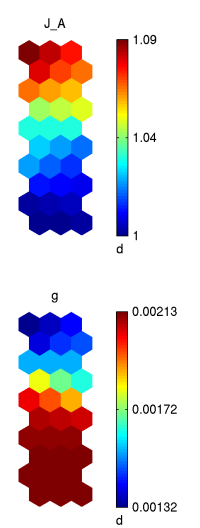
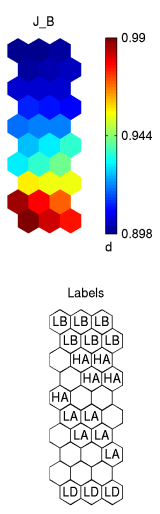

(b) Cost functions SOM.

Figure 13. SOM of the competitive game.

Cost functions SOM (Figure 13(b)) highlights the strong antagonism between drag and sonic boom, meaning that as one is minimized the other one is degraded. Furthermore a region across LA and HA configurations exists, thus providing an interesting trade-off between the two disciplines. Low-boom and high antagonism configurations show a strong nose deflection and an increase of the cabin radius. The acoustic function shows a strong influence of the nose deflection. As the antagonism is introduced by increasing $\epsilon$, the nose is rapidly deflected downward. At the same time the aerodynamic function is not impacted by this variable (see nose deflection SOM in Figure 14). For this reason it is the variable that shows the maximum rate of change since the beginning until the activation of the constraint. Low-drag configurations show a strong decrease of the dihedral angle, while $\mathrm{HA}$ and $\mathrm{LB}$ configurations have very different values ranging from -0.6 to -0.3 . Wing position and nose radius show a SOM strictly similar to that of the drag function. A low-drag configuration shows a smaller nose and a wing closer to the nose than other configurations.

Three configurations are retained for comparative analysis at different $\epsilon$ equal to $0,0.5$ and 1 . Main geometrical modifications are shown in Figure 15. When antagonism among disciplines increases first nose is deflected downward and then dihedral angle is increased. As $\epsilon$ increases, the nose shock is bounded in a region close to the nose top. The consequence is an increased nose shock amplitude, but more localized (Figure 16). The area under the first peak of the near field pressure signature is reduced as $\epsilon$ tends to 1 (Figures 17 and 18). This signature is directly related to the Whitham F-function, and in particular the first peak area is related to the nose shock amplitude and position. A reduced area determines a reduced front shock for the ground signature (Figure 18(b)).

The shock related to the wing leading edge is progressively increased as $\epsilon$ tends to 1 . The consequence is a reduction of the pressure expansion after the first peak of the ground signature as already seen in the analysis of the Pareto front obtained using the cooperative strategy. The low-pressure region on the bottom skin (Figure 12) at the trailing edge of the wing and at the corresponding junction with the fuselage is increased. The interaction with the aft shock pattern determines at ground a reduced amplitude of the rear shock. All the shocks in the ground signature contribute to the reduction of $J_{B}$. Nose shock overpressure is reduced by $2 \mathrm{~Pa}$ as well as the wing shock, while the aft shock shows a reduction by more than $4 \%$ from $\epsilon=0$ to $\epsilon=1$. 

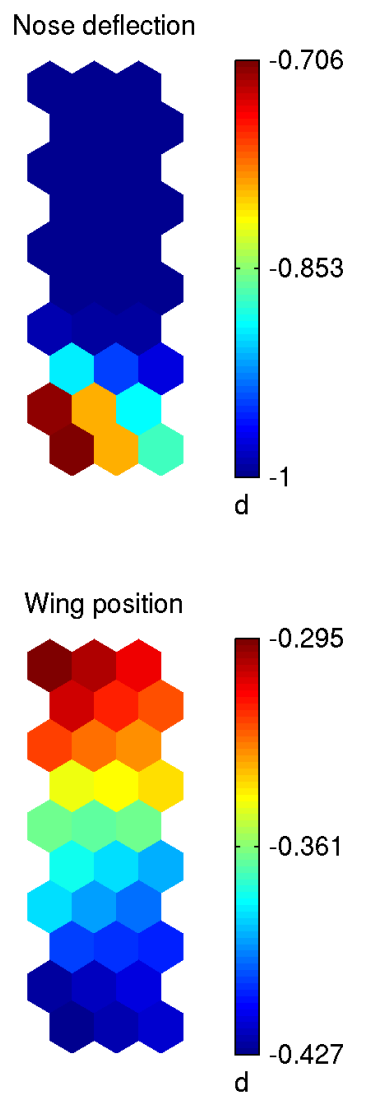

Nose radius

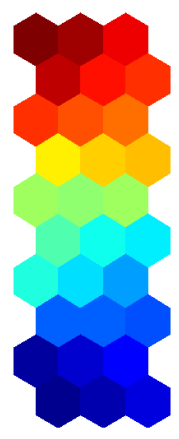

Dihedral angle

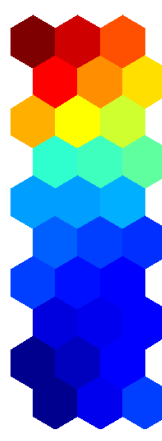

$-0.0891$

$-0.444$

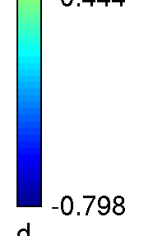

d

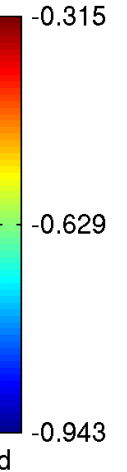

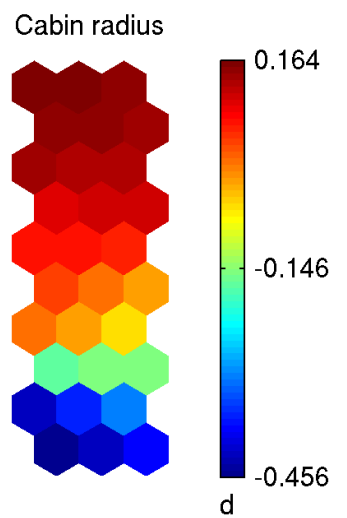

Labels

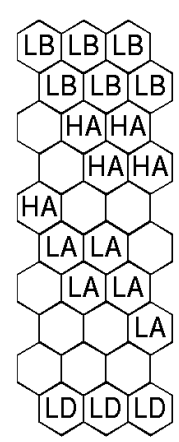

Figure 14. Variables SOM.

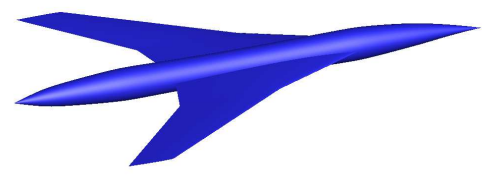

(a) Initial low-drag configuration
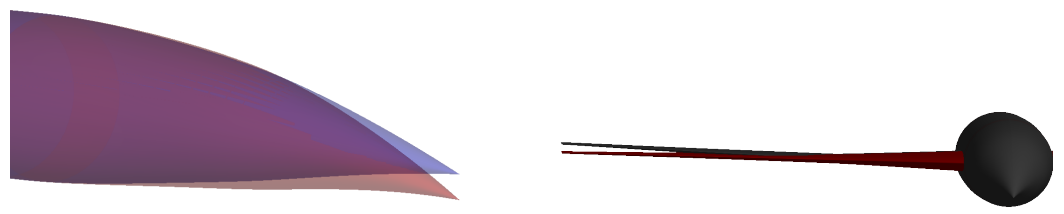

Figure 15. Main geometry modification during Nash game convergence. In blue the initial low-drag configuration $(\epsilon=0)$, in red the configuration at $\epsilon=0.5$, in black the configuration at $\epsilon=1$

\section{III.C. Coupling cooperation and competition}

The competitive phase provides a Nash equilibrium point that does not satisfy the Pareto-stationary condition. This means that it exists at least one point that shows the same acoustic performance but with a reduced drag. The coupling between a Nash game and a successive MGDA-CM phase will provide a solution that belongs to the Pareto front. Successive cooperation/competition iterations can provide a discretization of the Pareto front at a reduced computational cost and with diversity among non-dominated slutions defined by the user. The same surrogate models and parameterizations adopted for the competitive phase are 


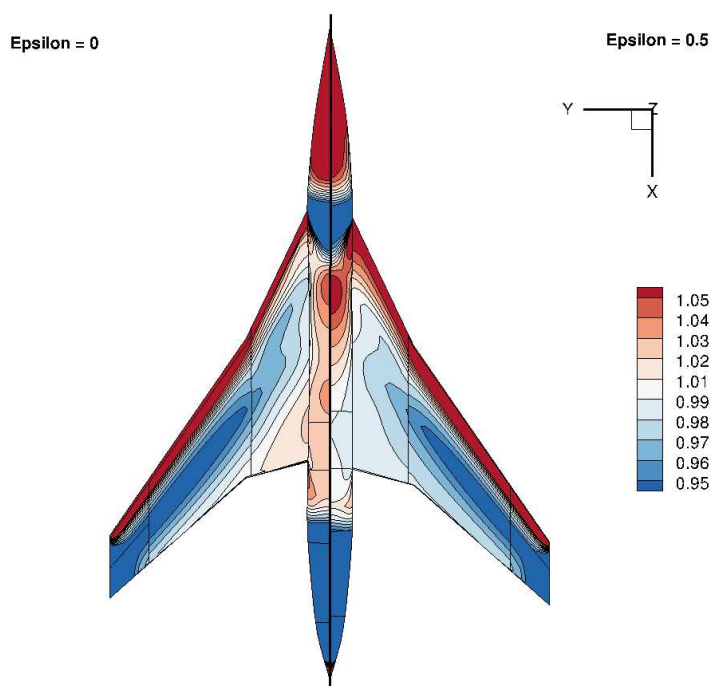

(a) Comparison between $\epsilon=0$ and $\epsilon=0.5$.
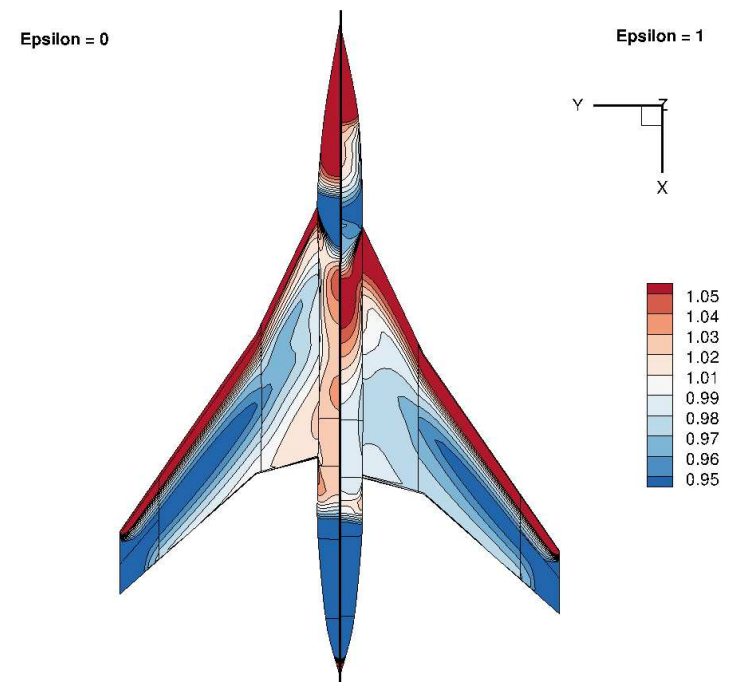

(b) Comparison between $\epsilon=0$ and $\epsilon=1$.

Figure 16. Bottom skin pressure field $\left(p / p_{0}\right)$ along the Nash equilibrium continuum.

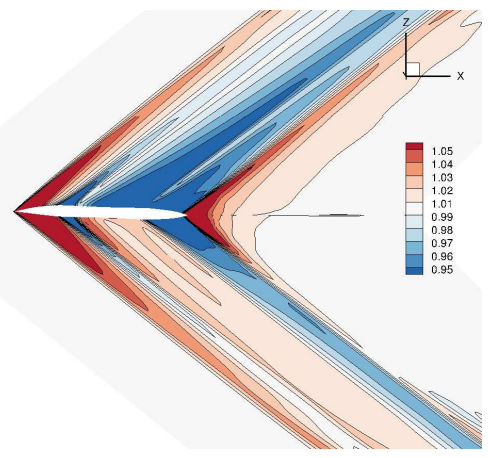

(a) $\epsilon=0$

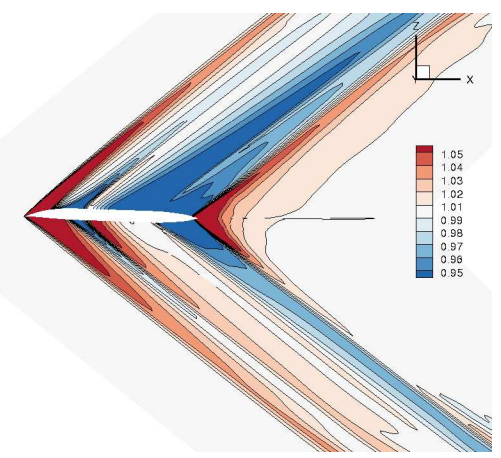

(b) $\epsilon=0.5$

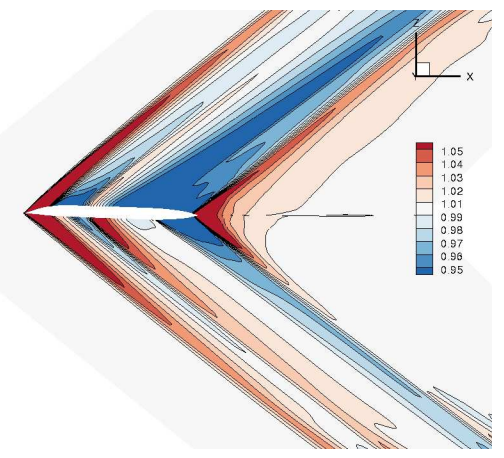

(c) $\epsilon=1$

Figure 17. Near-field pressure $\left(p / p_{0}\right)$ in the symmetry plane.

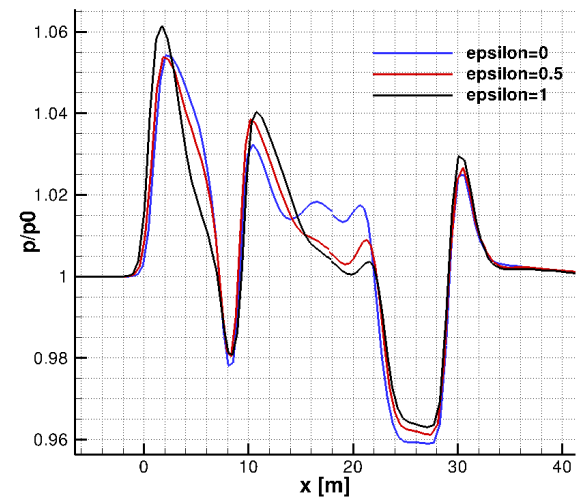

(a) Pressure signature $(p / p 0)$ extracted under-track at $\mathrm{z}=h_{Z}-10 \mathrm{~m}$

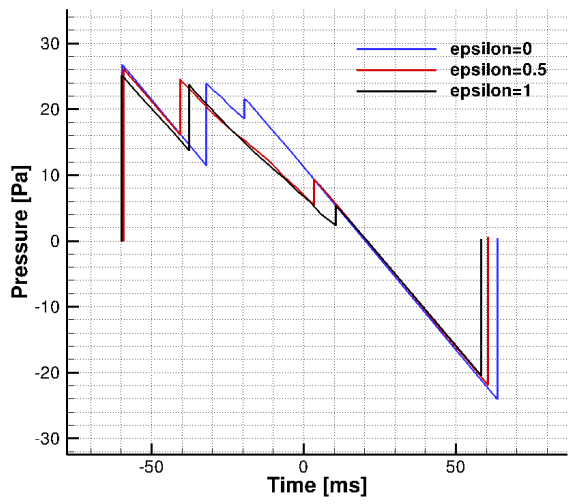

(b) Under-track ground signature

Figure 18. Near-field and ground pressure signal for different along the Nash equilibrium continuum.

considered here. The following steps are performed on Figure 19: 
- The minimization of the drag coefficient with constraint on lift and the competitive phase as in section III.B are performed providing respectively point 1 and 2 . In general it is useless to begin the competition phase when $\epsilon$ is equal to 1 , because the solution could be too far from the Pareto front and so not interesting for the designer. Despite this MGDA-CM is able to converge to the Pareto-stationary point also starting from $\epsilon=1$ (see Figure 18(a));

- A cooperative MGDA-CM optimization is performed providing point 3 on the Pareto front;

- A new $\alpha$ is evaluated in order to satisfy the Pareto-stationarity condition at 3;

- A new split matrix is evaluated using the Hessian of the function $J_{A}(\alpha)$ where $\alpha$ has been evaluated at the previous point of the process;

- Nash game is performed starting from the Pareto front in 3. The formulation adopted is written respectively written in equations (15) and (16);

- From point 4 a cooperative optimization is performed obtaining a point on the Pareto front 5 ;

- The previous four steps are repeated in order to obtain point 6 after Nash game iterations and point 7 after MGDA-CM convergence. The point 7 results close to the acoustic optimum.

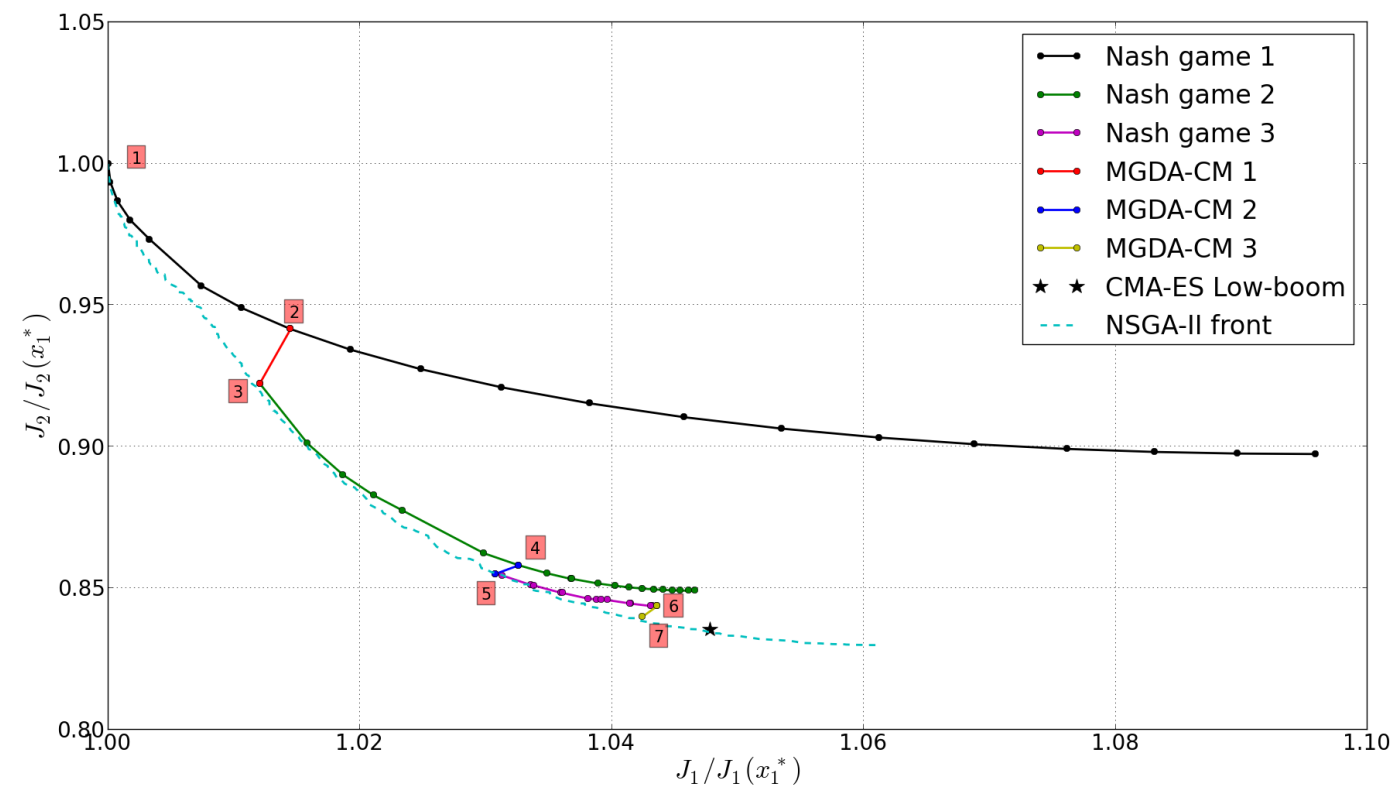

Figure 19. Coupling Nash games and MGDA iterations for the Pareto front exploration of the aero-acoustics problem.

In Figure 19 it is also shown the Pareto front obtained using NSGA-II based on a population of 24 individuals for 500 generations meaning approximatively ten times the total number of function evaluations required by the cooperative-competitive phase. In addition the Hessian based split provides Nash continuum curves that are tangent to the Pareto front at the starting Pareto-stationary point. The procedure can be repeated several times, also starting from different Nash equilibrium points. In the test case three coupling iterations have provided three points that belong to the Pareto front.

\section{Conclusions and perspectives}

Two innovative multi-objective optimization algorithms based on cooperation and competition have been described and applied to a low-boom/low-drag shape optimization problem of a SSBJ using either a highfidelity or low-fidelity models. 
The cooperation strategy is able to provide solutions that belong to the Pareto front, still avoiding an excessive number of function evaluations required by a classical multi-objective algorithm based on evolutionary strategies. Competition is able to introduce a trade-off between disciplines that is not associated with a severe deterioration of the primary or fragile ones. The coupling of the two strategies provides a promising methodology to explore the Pareto-set of optimal solutions at reduced computational cost compared to multi-objective stochastic optimization methods.

The application of these strategies to the aero-acoustic shape optimization of a supersonic business jet has provided the identification of several configurations thus defining a trade-off between the two discipline. Future work aims to improve both the disciplinary models, the parameterizations and the algorithms adopted. Concerning the model, the mixed-fidelity approach can be replaced by an high-fidelity model and the gradients and Hessian provided to the optimization algorithms using the adjoint method. As a consequence the curse of dimensionality problem of surrogate models can be avoided. An increase of the number of design variables will provide other set of possible configurations. Concerning the optimization algorithms, the MGDA algorithm can be enhanced introducing a scaling of the gradients inspired from Newton's method using Hessians.

\section{References}

\footnotetext{
${ }^{1}$ Marler, R.T. and Arora, J.S., "Survey of Multi-Objective Optimization Methods for Engineering," Structural and Multidisciplinary Optimization, vol. 26, no. 6, pp. 369-395, 2004.

${ }^{2}$ Miettinen, K., "Nonlinear Multiobjective Optimization," Springer, New York, 1999.

${ }^{3}$ Zitzler, E., and Thiele, L., "Multiobjective Evolutionary Algorithms: a Comparative Case Study and the Strength Pareto Approach," IEEE Trans Evol Comput, 3(4) pp. 25771, 1999.

${ }^{4}$ Zitzler, E., Deb, K., and Thiele, L., "Comparison of Multiobjective Evolutionary Algorithms: Empirical Results," Evol. Comput., 8(2):17395., 2000.

${ }^{5}$ Fonseca, C., and Fleming, P., "Multiobjective Optimization and Multiple Constraint Handling with Evolutionary Algorithms in a Unified Formulation." In: Transactions on Systems, Man and Cybernetics, Part A, vol. 28-1, pp. 26-37. IEEE., 1998.

${ }^{6}$ Knowles, J.D. , and Corne, D.W., "Approximating the Nondominated Front Using the Pareto Archived Evolution Strategy" Evol. Comput., vol. 8, pp. 149172, 2000.

${ }^{7}$ Zitzler, E., and Thiele, L., "An Evolutionary Algorithm for Multiobjective Optimization: The Strength Pareto Approach." Tech. Rep. 43, Computer Engineering and Networks Laboratory (TIK), Swiss Federal Institute of Technology (ETH) Zurich, 1998.

${ }^{8}$ Deb, K., Pratap, A., Agarwal, S., and Meyarivan, T., "A Fast and Elitist Multiobjective Genetic Algorithm: NSGA-II." IEEE Trans Evol Comput ;6(2):18297., 2002.

${ }^{9}$ Konak, A., Coit, D., and Smith, A., "Multi-objective Optimization using Genetic Algorithms: a Tutorial," Reliab. Eng. Syst. Saf., 91 (9), pp. 9921007, 2006.

${ }^{10}$ Tang, Z., Désidéri, J. A., and Périaux, J., "Multicriterion Aerodynamic Shape Design Optimization and Inverse Problems Using Control Theory and Nash Games",Journal of Optimization Theory and Applications, Vol. 135(3), pp. 599-622, 2007.

${ }^{11}$ Désidéri, J.A., "Cooperation and Competition in Multidisciplinary Optimization - Application to the aero-structural aircraft wing shape optimization", Computational Optimization and Application, Vol. 52, pp. 3-28, Springer Netherlands, 2012.

${ }^{12}$ Désidéri, J.A., "Multiple-Gradient Descent Algorithm (MGDA)". INRIA RR 6953, 2009. 2009.

${ }^{13}$ Zerbinati, A., Désidéri, J.-A., and Duvigneau, R., "Comparison between MGDA and PAES for Multi-Objective Optimization." Inria technical report RR-7667, 2011.

${ }^{14}$ Zerbinati, A., Désidéri, J.-A., and Duvigneau, R., "Application of Metamodel-Assisted Multiple-Gradient Descent Algorithm (MGDA) to Air-Cooling Duct Shape Optimization". In proceedings of ECCOMAS 2012.

${ }^{15}$ Vanderplaats, G.N., "CONMIN - A FORTRAN Program for Constrained Function Minimization: User's Manual", NASA TM X-62,282, 1973.

${ }^{16}$ Nash, J.F., "Non-cooperative Games", Ann. Math., vol. 54, pp. 286295, 1951.

${ }^{17}$ Periaux, J., Chen, H.Q., Mantel, B., Sefrioui, M. and Sui, H.T., "Combining Game Theory and Genetic Algorithms with Application to DDM-nozzle Optimization Problems," Finite Elements in Analysis and Design 37, pp. 417-429, 2001.

${ }^{18}$ Désidéri, J.-A., "Split of Territories", INRIA RR 6108, $200 \%$.

${ }^{19}$ Powell, M.J.D., "A Direct Search Optimization Method that Models the Objective and Constraint Functions by Linear Interpolation", in Advances in Optimization and Numerical Analysis, eds. S. Gomez and J-P Hennart, Kluwer Academic (Dordrecht), pp. 51-67,1994.

${ }^{20}$ Nelder, J.A., and Mead, R., "A Simplex Method for Function Minimization”. Comput. J. Vol. 7, No. 308., 1965.

${ }^{21} \mathrm{http}: / /$ docs.scipy.org/doc/scipy/reference/index.html

${ }^{22}$ Coulouvrat, F., "The Challenges of Defining an Acceptable Sonic Boom Overland," AIAA 2009-3384, 2009.

${ }^{23}$ Welge, H.R., Nelson, C., and Bonet, J., "Supersonic Vehicle Systems for the 2020 to 2035 Timeframe," AIAA 2010-4930, 2010.

${ }^{24}$ NASA LRC, "High-Speed Research: Sonic Boom, vol. I," NASA CP-3172, vol. 1, 1992.

${ }^{25}$ NASA LRC, "High-Speed Research: Sonic Boom, vol. II," NASA CP-3173, vol. 2, 1992.
} 
${ }^{26}$ Whitham, G.B., "The Flow Pattern of a Supersonic Projectile," Communications on pure and applied mathematics, Vol. 5, No.3, pp 301-348, 1952.

${ }^{27}$ Jones, L.B., "Lower Bounds for the Pressure Jumps of the Shock Waves of a Supersonic Transport of Given Length," Aeronaut. Quart., vol. XXIII, pt. 1, Feb. 1972, pp. 62-76. 20.

${ }^{28}$ Petty, L., "Lower Bounds for Sonic Boom Considering the Negative Overpressure Region", Journal of Aircraft,7,375-377, 1970.

${ }^{29}$ George, A.R., "Lower Bounds for Sonic Booms in the Midfield". AIAA J., vol. 7, no. 8, pp. 1542-1545, 1969.

${ }^{30}$ Seebass, R., and George, A.R. "Sonic-Boom Minimisation". Journal of the acoustical society of America, Vol 51 no.2 686-694. 1972

${ }^{31}$ Darden, C.M., "Sonic-Boom Minimization With Nose-Bluntness Relaxation", NASA TP-1348 1979.

${ }^{32}$ Rallabhandi, S.K., and Mavris, D.N. "Sonic Boom Minimization Using Inverse Design and Probabilistic Acoustic Propagation", Jo. of Aircraft Vol. 43, No. 6, NovemberDecember 2006.

${ }^{33}$ Plotkin, K., Rallabhandi, S., and Li, W., "Generalized Formulation and Extension of Sonic Boom Minimization Theory for Front and Aft Shaping", AIAA 2009-1052, 2009.

${ }^{34}$ Pawlowski, J.W., Graham, D.H., Boccadoro, C. H., Coen, P.G., and Maglieri, D.J., "Origins and Overview of the Shaped Sonic Boom Demonstration Program," AIAA Paper 2005-5, 43rd AIAA Aerospace Sciences Meeting and Exhibit, January, 2005.

${ }^{35}$ Honda, M., and Yoshida, K., "D-SEND Project for Low Sonic Boom Design Technology," ICAS 2012, 28th International Congress of the Aeronautical Sciences, September, 2012.

${ }^{36}$ Darden, C.M., "Limitations of Linear Theory for Sonic Boom Calculations," Journal of Aircraft, Vol. 30, No. 3 (1993), pp. 309-314.

${ }^{37}$ Choi, S., Alonso, J.J., Kroo, I., Wintzer, M., "Multifidelity Design Optimization of Low-Boom Supersonic Jets", J. Aircr. 45:10618, 2008.

${ }^{38}$ Minelli, A. , Salah el Din, I. and Carrier, G., "Advanced Optimization Approach for Supersonic Low-Boom Design." AIAA 2012-2168, 2012.

${ }^{39}$ Plotkin, K., and Page, J., "Extrapolation of Sonic Boom Signatures from CFD Solution." AIAA 2002-0922, 2002.

${ }^{40}$ Salah el Din, I., Le Pape, M.-C., Minelli, A., Grenon, R., and Carrier, G., "Impact of Multipole Matching Resolution on Supersonic Aircraft Sonic Boom Assesment." in proceedings of 4th EUCASS, St. Petersburg, 2011.

${ }^{41}$ Cambier, L., and Gazaix, M., "An Efficient Object-Oriented Solution to CFD Complexity." AIAA 02-0108, 2002.

${ }^{42}$ Bui, T.T., "CFD Analysis of Nozzle Jet Plume Effects on Sonic Boom Signature." NASA TM-2009-214650, 2009.

${ }^{43}$ Roe, P.L., "Approximate Riemann Solvers, Parameter Vectors and Difference Schemes", Journal of Computational Physics, 43, 357-372, 1981.

${ }^{44}$ Harten, A., Hyman, J.M. and Lax, P.D., "On Finite-Difference Approximations and Entropy Conditions for Shocks", Comm. Pure Appl. Math., 29, pp. 297-322, 1976.

${ }^{45}$ Van Albada, G.D., Van Leer, B., Roberts, W.W., "A Comparative Study of Computational Methods in Cosmic Gas Dynamics", Astron. Astrophysics 108: 7684, 1982.

${ }^{46}$ Taylor, A.D., "The TRAPS Sonic Boom Program." NOAA Technical memorandum ERL-ARL-87, 1980.

${ }^{47}$ Hansen, N., and Ostermeier, A., "Completely Derandomized Self-Adaptation in Evolution Strategies." Evolutionary Computation, 9(2) pp. 159195, 2001.

${ }^{48}$ Gill, P.E., Murray, W., and Wright, M.H., "Practical Optimization", Academic Press, London ISBN 0-12-283952-8, 1986.

${ }^{49}$ Kohonen, T., "Self-Organized Formation of Topologically Correct Feature Maps", Biological Cybernetics 43 (1): 5969, 1982. 